\title{
A new physiologically realistic and clinically relevant model of sleep apnoea for investigating it's effect as a comorbidity on neurodegenerative disease
}

\author{
Reno Roberts \\ University of Warwick \\ Mark Wall \\ University of Warwick \\ Ingke Braren \\ Institute for Experimental Pharmacology and Toxikology \\ Karendheep Dhillion \\ University of Warwick \\ Amy Evans \\ University of Warwick \\ Jack Dunne \\ University of Warwick \\ Simbarashe Nyakupinda \\ University of Warwick \\ Robert Huckstepp ( $\nabla$ R.Huckstepp@warwick.ac.uk) \\ University of Warwick https://orcid.org/0000-0003-4410-3397
}

Article

Keywords: sleep apnoea, co-morbidities, neurodegenerative disease

Posted Date: August 23rd, 2021

DOI: https://doi.org/10.21203/rs.3.rs-829873/v1

License: (c) (i) This work is licensed under a Creative Commons Attribution 4.0 International License.

Read Full License 
A new physiologically realistic and clinically relevant model of sleep apnoea for investigating it's effect as a comorbidity on neurodegenerative disease

3

\section{Running title: New model of sleep apnoea and cognitive decline}

Reno Roberts ${ }^{1}$, Mark J Wall ${ }^{1}$, Ingke Braren ${ }^{2}$, Karendheep Dhillion ${ }^{1}$, Amy Evans ${ }^{1}$, Jack Dunne ${ }^{1}$, Simbarashe Nyakupinda ${ }^{1}$, Robert T. R. Huckstepp ${ }^{1 *}$

\section{${ }^{1}$ School of Life Sciences, University of Warwick, Coventry, UK}

2 University Medical Center Eppendorf, Vector Facility, Inst. for Exp. Pharmacology and Toxikology, N30, Room 09, Martinistr. 52, 20246 Hamburg, Germany

\section{${ }^{*}$ Corresponding Author Email: R.Huckstepp@warwick.ac.uk}

\section{Abstract}

Sleep apnoea is a highly prevalent disease but often goes undetected and is associated with poor clinical prognoses when combined with many different disease states. However, most animal models of sleep apnoea (e.g., intermittent hypoxia) have recently been dispelled as physiologically unrealistic. Due to a lack of appropriate models, little is known about the causative link between sleep apnoea and it's co-morbidities. To overcome these problems, we have created a realistic animal model of moderate sleep apnoea by reducing the excitability of the respiratory network. This has been achieved through controlled genetically-mediated lesions to the preBötzinger Complex (preBötC), the inspiratory oscillator. This novel model shows increases in sleep disordered breathing with alterations in breathing during wakefulness (decreased frequency and increased tidal volume) as observed clinically. The increase in apnoea episodes leads to a reduction in REM sleep, with all lost active sleep being spent in an awake state. The increase in hypoxic and hypercapnia insults leads to both systemic and neural inflammation. Alterations in neurophysiology, an inhibition of hippocampal long-term potentiation (LTP), reflect deficits in both long and short term spatial memory. This new physiologically relevant and clinically realistic model of sleep apnoea may be the key to understanding why sleep apnoea has such far reaching and often fatal effects on end organ function.

\section{Introduction}

Current estimates of the prevalence of moderate to severe ( $\geq 15$ events. $\left.h^{-1}\right)$ sleep apnoea (SA: $>2$ missed breaths during sleep) are $\sim 4 \%$ of men and $\sim 2 \%$ of women in the UK. However, recent large cohort studies show that $91 \%$ of SA cases are undetected ${ }^{1}$, with an occurrence of $\sim 50 \%$ of men and $\sim 25 \%$ of women over $40^{2}$.

During Rapid Eye Movement sleep (REM), individuals experience increased upper airway resistance ${ }^{3}$ due to tonic motor inhibition lowering the muscle tone ${ }^{4}$. This leads to increased vulnerability to repetitive pharyngeal collapse and a cessation in airflow even in the presence of respiratory effort, otherwise known as obstructive sleep apnoea (OSA) $)^{5}$. During non-Rapid Eye Movement sleep (NREM), a loss of descending inputs into the respiratory centres, means control of breathing is solely down to metabolic factors, and breathing is more reliant on chemoreception ${ }^{4}$. Thus any defects in the respiratory microcircuit will be exposed leading to a loss of drive to the respiratory muscles, termed central sleep apnoea (CSA) ${ }^{4}$. Whilst it is believed that OSA is a REM related disordered with CSA is linked to NREM, both CSA and OSA occur during either NREM or REM sleep ${ }^{6,7}$. In all cases, patients experience intermittent hypoxic hypercapnia following cycling periods of apnoea and hyperpnoea ${ }^{8}$.

The blood/gas changes lead to oxidative imbalance facilitating the generation and build-up of reactive oxygen species (ROS) leading to the development of systemic inflammatory-related biomarkers associated with chronic inflammation ${ }^{9}$. Molecular markers of systemic inflammation are increased in patients with $\mathrm{SA}^{10}$, leading to an increased risk of diseases with an underlying inflammatory component, e.g., cardiovascular disease (CVD $)^{11}$, diabetes ${ }^{12}$, and dementia ${ }^{13}$. Systemically, this can lead to heart failure $(\mathrm{HF})^{14}$, with mortality directly related to $\mathrm{SA}$ severity ${ }^{15}$ and $\mathrm{SA}$ treatment improving 
mortality ${ }^{16}$ and morbidity ${ }^{17}$. As SA causes endothelial dysfunction through inflammation, and endothelial dysfunction is capable of impairing cerebral blood perfusion ${ }^{18}$. In addition, an increase in hypertension ${ }^{19}$ and atherosclerosis ${ }^{20}$ in SA patients leads to recurrent strokes ${ }^{21}$. Furthermore, the hypercapnic hypoxia leads directly to oxidative stress and neuroinflammation ${ }^{22,23}$. All of these brain insults individually or in combination lead to decreased hippocampal and grey matter volume ${ }^{22,23}$ resulting in memory deficits, and cognitive decline ${ }^{13,23}$.

Current animal models of SA used to study these pathways and associations are severely flawed: Chronic intermittent hypoxia, replicates the hypoxia observed during SA, but increased compensatory breathing leads to hypocapnia, the opposite of the clinical condition. Airway occlusion replicates the blood gases changes that occur during apnoea, but the frequency and duration of the apnoeas do not replicate the clinical presentation. In both of these models the simulated "apnoeas" continue after arousal or occur during wakefulness, which initiates the defence response which is physiologically unrealistic, and will contribute to any of the related comorbidities. Here we have developed a physiologically realistic model of SA, that only displays apnoeas during natural sleeping patterns. Furthermore, our model displays both systemic and neural inflammation and exhibits cognitive deficits, making it clinically relevant. This novel physiologically realistic and clinically relevant model of moderate sleep apnoea can be utilised for investigating the effect of SA on associated conditions such as neurodegenerative disease

\section{Results}

AAV:Syn-DTA (DTA) transduction of the preBotC leads to a lesion of rhythm generating neurons Previously, substance-P conjugated to saporin (SP-SAP) was used to create progressive loss of neurokinin 1 receptor positive neurons ${ }^{24}$. When SP-SAP is injected into the inspiratory oscillator, the preBötzinger Complex (preBötC), it leads to sleep apnoea once the lesion size reaches $\sim 25 \%$ of the NK1R neurons ${ }^{24}$. Unfortunately as lesions using this neurotoxin increase over time, the breathing issues soon progress into wakefulness ${ }^{24}$, meaning this can not be used as an accurate model of sleep apnoea for chronic studies. We have therefore taken a different approach and created a stable partial lesion of the preBötC, which led to a $22 \%$ loss of neurons (Sham: $1614 \pm 316$ neurons, $n=11$ vs DTA: $1264 \pm 378$ neurons, $n=8 ; p=0.04$; Fig $1 B$ ). This was achieved by transfection with an adenoassociated virus (AAV) leading to expression of diphtheria toxin sub unit-A (DTA; Figs. 1A-B,7A). This represented the loss of a significant portion of rhythm generating cells (Sham: $40 \pm 19$ NK1R intensity, $n=11$ vs DTA: $24 \pm 7$ NK1R intensity, $n=7 ; p=0.04$; Fig $1 A$ ).

DTA lesions lead to sleep disordered breathing and alterations in respiration during wakefulness.

As viral transduction led to a significant lesion of the preBotC, we next studied how this effected breathing and whether this led to sleep apnoea. Breathing of rats was measured with plethysmography (Fig. 1C, 7A) and sleep-wake state via EEG/EMG recordings (Fig. 2A,B, 7B-C). For assessment of SA severity, we measured the apnoea-hypoxia Index (AHI: sum of apnoeic + hypopnoeic events per hour of sleep), as is done clinically. AHI was increased in DTA injected rats (Sham: $18 \pm 4$ incidences/hour of sleep, $n=13$ vs DTA: $52 \pm 16$ incidences/hour of sleep, $n=11 ; P=0.0000006$ ), as was total duration of apnoeas + hypopnoeas (Sham: $47 \pm 13$ secs, $n=13$ DTA: $101 \pm 37$ secs, $n=11 ; p$ $=0.00004$; Fig 1E). Apnoeas occurred during: 1) NREM sleep (Fig 1C) and REM sleep (Fig 1C). Rats with preBötC lesions had an increased $f$ (Sham: $95 \pm 7$ breaths/min, $n=14$ vs DTA: $105 \pm 14$ breaths/min, $n$ $=11 ; p=0.02$; Fig 1D ), maintaining $V_{e}$ (Sham: $343 \pm 163 \mathrm{~mL} / \mathrm{kg}, \mathrm{n}=14$ vs DTA: $244 \pm 129 \mathrm{~mL} / \mathrm{kg}, \mathrm{n}=$ 11; $n s ;$ Fig 1D) through a reduction in $V_{T}$ (Sham: $3.6 \pm 2 \mathrm{~mL} / \mathrm{kg}, \mathrm{n}=14$ vs DTA: $2.3 \pm 1 \mathrm{~mL} / \mathrm{kg}, \mathrm{n}=11 ; \mathrm{p}$ $=0.03$; Fig 1D).

Rats with sleep apnoea have reduced REM sleep with no effect on time spent in NREM.

Given the increase in apnoeas observed during both phases of sleep, and apnoeas induce microarousals, we tested whether our DTA injected rats exhibited any alterations in sleeping patterns. DTA lesions led to the onset of sleep disturbances (Fig 2). Whilst there was no effect on the percentage of time spent in NREM sleep (Sham: $48 \pm 4 \%, n=14$ vs DTA: $46 \pm 6 \%, n=11 ; p=0.4$; Fig 2 A-C), arousals 
caused by apnoeic events decreased the time rats spent in REM sleep by 30\% (Sham: $6 \pm 1$ breaths/min, $n=14$ vs DTA: $4 \pm 1$ breaths/min, $n=11 ; p=0.003$; Fig $2 A-C)$; with all of this lost REM time being spent awake (Sham: $46 \pm 5 \%, n=14$ vs DTA: $52 \pm 6$ breaths/min, $n=11 ; p=0.01$; Fig $2 A$ C).

Rats with sleep apnoea have alterations in systemic inflammatory markers and plasma cateacholamine levels

Hypercapnic-hypoxia leads to chronic inflammation ${ }^{9}$, in SA patients ${ }^{10}$. Therefore, we explored inflammatory markers from inferior vena cava blood samples. Our cytokine panel showed rats with sleep apnoea displayed increased levels of IFNY (Sham: $2 \pm 0.3, n=11$ vs DTA: $2.8 \pm 0.7, n=9 \mathrm{ng} / \mathrm{mL}$; $\mathrm{p}=0.003$ ), IL-1b (Sham: $0.6 \pm 0.1, \mathrm{n}=12$ vs DTA: $0.8 \pm 0.1, \mathrm{n}=8 \mathrm{ng} / \mathrm{mL} ; \mathrm{p}=0.049$ ), and MIP (Sham: 14 Range $_{70}=1 \mathrm{ng} / \mathrm{mL}, \mathrm{n}=7$ vs DTA: 336 Range $_{70} 170 \mathrm{ng} / \mathrm{mL}, \mathrm{n}=9 ; \mathrm{P}=0.0009 ;$ Fig 3 ) in SA rats. With no change in plasma levels of IL- 1 a (Sham: $1.9 \pm 0.1, n=12$ vs DTA: $1.9 \pm 0.1, n=9 \mathrm{ng} / \mathrm{mL} ; p=0.96$ ), IL-6 (Sham: $1.2 \pm 0.0, n=12$ vs DTA: $1.2 \pm 0.0, n=8 \mathrm{ng} / \mathrm{mL} ; \mathrm{p}=0.6$ ), TNFa (Sham: 16 Range $_{70}=9.8 \mathrm{ng} / \mathrm{mL}$, $\mathrm{n}=13$ vs DTA: 16 Range $_{70} 8.2 \mathrm{ng} / \mathrm{mL}, \mathrm{n}=9$; $\mathrm{P}=0.5$; Fig 3), MCP-1 (Sham: 4.0 Range $_{70}=0.1 \mathrm{ng} / \mathrm{mL}, \mathrm{n}=$ 12 vs DTA: 4.0 Range $_{70} 0.1 \mathrm{ng} / \mathrm{mL}, \mathrm{n}=9 ; \mathrm{P}=0.9$; Fig 3), Rantes (Sham: 2.1 Range $_{70}=0.1 \mathrm{ng} / \mathrm{mL}, \mathrm{n}=10$ vs DTA: 2.3 Range $_{70} 0.5 \mathrm{ng} / \mathrm{mL}, \mathrm{n}=9 ; \mathrm{P}=0.3$; Fig 3). Showing a very specific inflammatory panel in our model of sleep apnoea. Furthermore, we found our model of sleep apnoea displayed increased plasma noradrenaline (Sham: $9.90 \pm 0.06 \mathrm{ng} / \mathrm{mL}, \mathrm{n}=8$ vs DTA: $9.94 \pm 0.007 \mathrm{ng} / \mathrm{mL}, \mathrm{n}=9 ; \mathrm{p}=0.04 ;$ Fig 2D), a marker of increased sympathetic output seen in sleep apnoea patients ${ }^{25}$.

\section{Rats with sleep apnoea have neuroinflammation}

As $\mathrm{IL}-1 \mathrm{~b}$ was elevated in $\mathrm{SA}$ rats, and $\mathrm{IL}-1 \mathrm{~b}$ is intimately linked to microglial activation ${ }^{26}$ and neuroinflammation ${ }^{27}$, we investigated Iba1 (a marker of activated microglia) levels in the hippocampus. SA rats displayed neuroinflammation in the hippocampus, which had elevated levels of activated microglia in the CA1 region (Sham: 3 Range $_{70} 20$ cells, $n=21$ vs DTA: 21 Range $_{70} 4$ cells, $n=$ 7: $p=0.007$; Fig $4 A, B$ ) dentate gyrus (Sham: $5 \pm 3$ cells, $n=10$ vs DTA: $11 \pm 3$ cells, $n=7: p=0.001$; Fig. $4 C, D)$.

\section{Rats with sleep apnoea have neurophysiological changes resulting in reduced LTP}

We next tested to see if the neuroinflammation in SA rats altered the electrophysiological properties in the hippocampus. We first investigated basal synaptic transmission in area CA1 of the hippocampus (Schaffer collateral-CA1 synapses, SC-CA1). There was no difference in the stimulus input-output curves between SA and sham rats (mean difference $-18.9 \pm 12.2 \mathrm{mV} / \mathrm{ms}, \mathrm{p}=0.1$; fig. $4 \mathrm{~A}$ ), and also no significant difference in the paired pulse ratio (mean difference $0.0009 \pm 0.04, p=0.98$; fig. 4B). Thus, basal synaptic transmission appears unaffected by apnoea. To investigate long term potentiation (LTP) we used theta burst stimulation (TBS, see methods). SA rats showed decreased LTP following theta burst stimulation. Both shams (mean difference -0.6; std error 0.1, DF $=26 ; p=0.00001$ ) and SA rats (mean difference -0.5; std error 0.1, DF $=26 ; p=0.00003$ ) showed a significant short term potentiation immediately following TBS (Fig 4C,D). However, the short term potentiation was not converted into long term potentiation in SA rats as the change in slope was reduced after 1 hour (mean difference 0.3 ; std error $0.8, D F=26 ; p=0.01$ ) and was no longer different to baseline (mean difference $-0.2 ;$ std error 0.1, DF $=26 ; p=0.4$; Fig $4 C, D$ ). In contrast, the sham rats displayed LTP, with their change in slope remaining elevated at a level not different to that seen immediately after TBS (mean difference -0.2; std error 0.1, DF = 26; $p=0.4$ ) and higher than baseline (mean difference 0.2; std error 0.1, DF = 26; $p=0.005 ;$ Fig $4 C, D)$. Thus SA rats exhibited neurophysiological deficits. which could result in cognitive impairment

Rats with sleep apnoea have deficits in short term memory

Neuroinflammation contributes to cognitive impairments through the depletion of synapses and their plasticity ${ }^{28}$. We sought to assess how the neuroinflammation we observed in our SA rats affected learning and memory. In a Y-maze forced alteration test (short term memory; tests separated by 1 hour), rats with SA displayed no preference for the novel arm in terms of Entries into ( $54 \% \pm 4 \%$; mean 
difference -7 ; std error $5, D F=14 ; p=1)$, Duration in $(52 \% \pm 5 \%$, mean difference -4 ; std error $5, D F=$ $14 ; p=0.7)$ and distance travelled $(53 \% \pm 4 \%$; mean difference 7 ; std error $4, D F=14 ; p=0.96)$ in the novel vs open arm (Fig. $6 \mathrm{~A}-\mathrm{B}$ ). In contrast shams displayed a strong preference for the novel are (Entries: $61 \% \pm 4 \%$, mean difference 22; std error 6 , DF $=14 ; p=0.02$; duration: $57 \% \pm 5 \%$, mean difference 22; std error 7, DF $=13 ; \mathrm{p}=0.046$; and distance travelled: $61 \% \pm 3 \%$, mean difference 22; std error $5, D F=14 ; p=0.004$; Fig. $6 A-B)$. This was not confounded by anxiety or locomotor issues as rats covered the same distance (Sham: $15 \pm 5 m, n=7$ vs DTA: $17 \pm 5 m, n=9 ; p=0.4$; not shown).

\section{Rats with sleep apnoea have deficits in long term memory}

Whilst the SA rats displayed impaired short term memory, we next investigated whether they also showed deficits in the conversation of short to long term memory. Rats with SA spent longer investigating a Barnes maze in terms of duration (Sham: $31 \pm 25$ vs DTA: $189 \pm 22$ secs; mean difference 158; std error 35; $D F=14 ; p=0.00002$ ) and distance covered (Sham: $2.4 \pm 1.5$ vs DTA: $10.6 \pm 1.3 \mathrm{~m}$; mean difference -8 ; std error 2; $D F=14 ; p=0.0008$, Fig $6 C-D)$. SA rats failed to use the escape hole more often (Sham: $7 \pm 1$ vs DTA: $4 \pm 2$, mean difference -10 ; std error 2 ; DF $=14 p=0.0002$; Fig 6C-D). Rats with SA were less likely to utilise spatial cues (Sham: $48 \%$ vs DTA: $29 \%$; mean difference 1.8; std error $0 ; p<0.0001$ ) instead preferring a sequential search strategy (Sham: $50 \%$ vs DTA: $61 \%$; mean difference -0.6 ; std error $0 ; p<0.0001)$, with an almost 5 times as many rats utilising a random search strategy (Sham: $2 \%$ vs DTA: $10 \%$; mean difference -0.75 ; std error $0 ; p<0.0001$ ) search strategy (Fig $6 \mathrm{E})$.

\section{Discussion}

Recently sleep apnoea has been gathering attention. It's far-reaching effects as a comorbidity for many diseases makes it a promising target for understanding the mechanisms of those diseases, as well as providing new therapeutic targets. The major issue is that, until now, there have been no good models for studying sleep apnoea.

We created a stable lesion of the inspiratory oscillator using a virus to transduce cells to produce the alpha subunit of diphtheria toxin (DTA). DTA inhibits protein synthesis ${ }^{29}$ leading to apoptosis of the transduced neurons. Following apoptosis, DTA cannot enter neighbouring cells in the absence of the $B$ subunit ${ }^{30}$. This creates a stable lesion limited only to the transduced cells. If the lesion size is restricted, then loss of respiratory rhythm generating neurons in the preBötC should be compensated during wakefulness ${ }^{24}$. However, during sleep a reduction in chemosensory drive and inhibition of afferent inputs to the respiratory system, makes breathing more fragile leading to apnoeas ${ }^{24}$. Transduced rats developed sleep disordered breathing, that closely resembles the breathing patterns observed in SA patients. Apnoeas occurred during NREM sleep (Fig 1C) when breathing becomes more dependent on medullary nuclei and REM sleep (Fig $1 \mathrm{C}$ ) likely due to decreased inspiratory drive to the tongue exacerbating nocturnal muscle paralysis leading to airway collapse. Furthermore, the respiratory changes during wakefulness in rats with sleep apnoea were similar to the clinical presentation where untreated SA patients display increased $f$ with a reduction in $\mathrm{V}_{T}$, thus maintaining $\mathrm{V}^{31}$.

In addition, to the respiratory similarity we found sleep-wake disturbances in our model mimic the disease. REM sleep in OSA patients with and without CPAP showed no change in NREM sleep (CPAP: $68 \%$ vs OSA $68 \%$ ), but saw a $26 \%$ reduction in REM sleep in untreated OSA patients (CPAP: $16 \%$ vs OSA $9 \%$ ), with all of the lost REM time being spent awake (CPAP: $16 \%$ vs OSA $22 \%$ ) (numbers calculated from table 1 in ${ }^{32}$ ), the same trend we observed in our rats. Alterations in REM sleep are directly linked to cognitive decline ${ }^{33}$, and REM sleep disturbances are now thought to be an extremely reliable biomarker for $A D^{34}$. This sleep deprivation will contribute to a decrease in cognition as REM is crucial for memory consolidation ${ }^{35}$

Remarkably, we found only a select panel of inflammatory markers elevated in the plasma of our moderate sleep apnoea model, IFNY, IL-1 $\beta$, and MIP. IFN $\gamma$ activates interferon regulatory factor 1 $(\text { IRF1 })^{36}$ through stimulation of STAT ${ }^{37}$ (Fig. 7), with an $800 \%$ increase in IRF1 following IFNy induced 
M1 polarisation of macrophanges ${ }^{38}$. IFR1 induces transcription of caspase 1 , which cleaves pro-IL-1 $\beta$ to produce the active IL-1 $\beta$ protein ${ }^{39}$ (Fig. 7); therefore modulation of IL-1 $\beta$ is via post transcriptional modification rather than increased transcription, hence IL-1 $\beta$ protein is increased even though paradoxically it's mRNA is diminished. Caspase 1 further aids IL-1 $\beta$ release, by cleaving Gasdermin D (GSDMD) to form the pore responsible for IL-1 $\beta$ release ${ }^{39}$ (Fig. 7). Once released, IL-1 $\beta$ induces the secretion of MIP2 and MIP1 $\beta$ from paranchemyal cells ${ }^{40}$. In parallel, IFNy induces both MIP1 $\alpha$ and MIP1 $\beta$ release from macrophanges ${ }^{41}$, likely in a caspase 1 dependent manner ${ }^{42}$ (Fig 7).

Interestingly, whilst IFNy activation leads to IL-1 $\beta$ and MIP secretion, it cannot by itself induce release of IL- $6^{41}$, RANTES ${ }^{43}$, or MCP- ${ }^{44}$. Given that IL- 6 is responsible for inducing CRP release from the liver ${ }^{45}$, it is therefore not surprising that plasma levels of CRP also remained unchanged. In conjunction, whilst IFN $\gamma$ activated macrophages release TNF ${ }^{46}$, this is inhibited by hypercapnia ${ }^{47}$, hence TNF $\alpha$ would not be expected to be elevated in response to moderate sleep apnoea. Finally, cleavage of pro-IL-1 $\alpha$ to IL$1 \alpha$, occurs through a calpain dependent, caspase independent, pathway ${ }^{39}$. Given calpain is inhibited by oxidative stress ${ }^{48}$, whilst caspase 1 is activated by it ${ }^{49,50}$, would provide a basis for why IL-1 $\alpha$ and IL$1 \beta$ are differentially regulated during sleep apnoea.

Hypoxia plays an important role in sterile information. Lowered $\mathrm{O}_{2}$ increases plasma IFNy ${ }^{51}$, and leads to toll like receptor 4 (TLR4) $)^{52}$ and MIP1a receptor ${ }^{53}$ upregulation and activation. The increased IFNy leads to IRF1 dependent inflammation which is enhance by hypoxia induced ROS stimulation of the TLR4 pathway ${ }^{54}$. Furthermore, upregulation of $\mathrm{MIP}^{55}$ and $\mathrm{TLR}^{56}$ will enhance caspase 1 activity through NFKB activation, and amplifying cleavage pro-IL-1 $\beta$ to it's active form ${ }^{49}$ (Fig 7). Hence IFNy-, IRF1-, Caspase-, TLR4-, and MIP- all drive microgliosis ${ }^{49,57}$. Co-activation of TLR4 and the IFNy receptor leads to significant neuronal dysfunction and cell death ${ }^{58}$. With neuronal cell death increased by hypoxia induced caspase activity ${ }^{50}$. Therefore hypoxia, leads to IFNy induced microglial activation which stimulates IRF1 to induce caspase activity to cleave pro-IL-1 $\beta$, a pathway enhanced by activation of TLR4 and MIP1 $\alpha$ by ROS (Fig 7), leading to microgliosis, and neuronal damage. These effects on neural inflammation may be why, IFNy is inversely correlated with cognitive ability ${ }^{59}$ and grey matter volume in the dorsal hippocampus ${ }^{59}$, and may therefore contribute to grey matter loss and mild cognitive impairment (MCI) in SA patients ${ }^{60}$.

Microglial activation induces tau phosphorylation ${ }^{61}$ in neurons, and leads to conversion of $\mathrm{MCl}$ to both $A D$ and $V a D^{62}$. In addition to Tau phosphorylation sleep apnoea also contributes to Alzheimer's disease (AD) through greater amyloid burden ${ }^{63}$. Sleep apnoea is independently associated with increased dementia and $\mathrm{MCl}$ such as reduced memory and executive function ${ }^{64,65}$, with executive function being more vulnerable than memory, or core intellectual and verbal abilities ${ }^{66,67}$. With treatment of sleep apnoea being able to partial reverse this cognitive decline ${ }^{68,69}$. The loss of REM sleep, the activation of microglia, and the direct effects of inflammation on hippocampal size and function, may be why sleep apnoea has such profound effects on the brain, and the deficits of long and short term memory we saw in our rats.

In summary we have developed a new model of moderate sleep apnoea that exhibits all of the clinical presentations tested. This model will be a valuable a new research tool for investigation of sleep apnoea directly and as a comorbidity for other disorders such as neurodegeneration.

\section{Acknowledgements}

RR: performed experiments and analysed data from all aspects of the project. MJW: Performed LTP experiments, designed LTP experiments, helped design Barnes maze experiments, and helped write the manuscript. IB: Designed and made the virus. KD performed plethysmography and ELISAs, and analysed ELISA data. AE: Performed plethysmography and ELISAs. JD: performed plethysmography, Barne's maze, and Y-maze experiments. SN: Performed immunocytochemistry. RTRH: Designed and performed experiments and analysed data from all aspects of the project, and oversaw the project. 
All authors commented on the manuscript. We would also like to thank Prof. Bruno Frenguelli for use of his equipment and for his consultation during the early experimental design of the LTP experiments.

\section{Methods}

rAAV Vector particle production and purification

rAAV were produced in Sf9 insect cells. The rAAV transfer plasmid were derived from pFBGR (kindly provided by Robert Kotin, National Heart, Lung and Blood Institute, National Institutes of Health, Bethesda, MD, USA). InFusion HD Cloning Kit (Clontech) was used according to the recommendations of the manufacturer for two-fragment reactions. Diphteria toxin fragment A (DTA) followed by an IRES and GFP as a fluorescent marker was inserted into pFBGR under control of a human synapsin promoter by InFusion cloning. Two fragments were generated by PCR using PrimeStar GLX Polymerase (Clontech): The human synapsin promoter was amplified from pAAV-Syn-Venus (Tang et al., The Journal of Neuroscience, July 8, 2009) using 5'-tctagaaatattaaggtacgggaggtacttgg and 5'GATCcttgctagcagcttgaattcgactgcgctctca. DTA-IRES-GFP was amplified from pAAV-DTA-IRES-GFP (kindly donated by Prof. Alex Gourine, UCL) using 5'ctgctagcaagGATCcaccATGGATCCTGATGATGTTGTTG and 5'gggcgaattgggtaccTTACTTGTACAGCTCGTCCATGC. pFBGR-Ultra GFP was cut with Mlul and Kpnl and both fragments were inserted simultaneously using the InFusion HD Cloning Kit (Takara Clontech) according to the recommendations of the manufacturer to generate the AAV transfer plasmid pFBGRUltra Syn DTA-IRES-GFP. Final AAV transfer plasmids were confirmed by restriction digest, by PCR using the specific primers mentioned above and by sequencing.

For packaging of AAV serotype 1 particles, pSR651 (Addgene plasmid 65213) was employed. For packaging of AAV serotype 2 particles, pSR657 (Addgene plasmid 65214) was employed (Smith et al. Molecular Therapy vol. 17 no. 11 nov. 2009). For producing rAAV serotype 9 particles, pFastBacDual (Life Technologies) was employed and rep2 cap9 were introduced according to Chen et al., Molecular Therapy vol. 16 no. 5 may 2008.

To monitor Sf9 transduction efficiency during rAAV production, GFP was introduced into pFBGR under control of baculoviral late basic promoter (pB) and mCherry was introduced into pSR651 and pSR657 generating pFBGR-Ultra GFP and pSR651-Ultra mCherry and pSR657-Ultra mCherry ("Ultra-Bac", Philipps et al. 2005). pB was amplified from Baculovirus Genomic DNA using 5'GCAATTGTTGTTGTTAAATTCCGTTTTGCGACGATGC and GTTTAAATTGTGTAATTTATGTAGCTG), GFP was amplified from pscAAV-GFP and mCherry was amplified from pSicoR-Ef1a-mCh-Puro-GFPi (Addgene plasmid 31848) using 5'-TTACACAATTTAAACGctagcATGGTGAGCAAGGGCGAGG and 5'tgcaataaacaagttaacTTACTTGTACAGCTCGTCCATGC).

Baculovirus genomic DNA carrying AAV genomic components and packaging elements was produced according to the Bac-to-Bac Expression System (Life Technologies) according to the recommendations of the manufacturer. E.coli DH10Bac were transformed and colonies were grown on Kanamycin/Tetracyclin/Gentamycin plates supplemented with IPTG/Bluo-Gal. Large white colonies were propagated in $5 \mathrm{ml}$ 2YT medium containing Kanamycin/Tetracyclin/Gentamycin overnight. Baculoviral DNA was extracted by Miniprep alkaline lysis, precipitated with 2-propanol and resuspended in $50 \mu \mathrm{lddH_{2 }}$ O. Clones were verified by PCR using Synapsin/T7 promoter specific primers.

rAAV were produced according to the "titerless infected-cells preservation and scale-up (TIPS)" also known as Baculovirus-infected insect cells (BIIC) (Wasilko et al., Protein Expr Purif. 2009 Jun;65(2):122-32.). Therefore, Sf9 cells were seeded in 12 well plates and baculovirus DNA derived from pFBGR-Ultra Syn DTA-IRES-GFP, pSR651-Ultra mCherry, pSR657-Ultra mCherry and pFastBacDual-Ultra rep2 cap9 were transfected with the recombinant baculoviral genomic DNA using TransIT-Insect (MoBITec) according to the recommendations of the manufacturer. After 3 days, transfection could be monitored by mCherry or GFP fluorescence. Sf9 cells were collected and 2E+07 fresh Sf9 cells were infected with this primary stock. After 1 day, vital fluorescing Sf9 cells were collected and frozen at $2 \mathrm{E}+6$ cells BIIC stocks in InsectXpress/10\% DMSO. 
For production of $\mathrm{rAAV}, 4 \mathrm{E}+08$ fresh Sf9 cells (Merck) were cultivated in InsectXpress Medium (Lonza) at a density of 1-2E+6 cells $/ \mathrm{ml}$. $~ 2 \mathrm{E}+06$ Sf9 BIIC stocks containing the expression cassette Syn DTAIRES-GFP and $\sim 2 E+06$ Sf9 BIIC stocks containing the desired AAV packaging elements were added. After 4 days, cells were harvested and spun down for $20 \mathrm{~min}$ at 2,000 $\mathrm{x}$ g. The cell pellet was

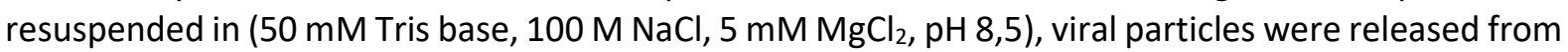
the nucleus by three freeze-thaw cycles. Virus was precipitated by incubation overnight in $10 \%$ PEG$8000 / 1 \mathrm{M} \mathrm{NaCl}$ at $4^{\circ}$ and then centrifuged for $30 \mathrm{~min}$ at $4^{\circ}$ and 3,000 $\mathrm{xg}$. The resulting pellet was combined with cell lysates. Benzonase (Merck KGaA, Darmstadt, Germany; final concentration of 250 $\mathrm{U} / \mathrm{ml}$ ) was added and incubated for $1 \mathrm{~h}$ at $37^{\circ} \mathrm{C}$. Cell debris was pelleted for $20 \mathrm{~min}$ at $12,000 \times \mathrm{g}$ and vector containing lysates were purified using lodixanol step gradients. $40 \%$ lodixanol layers were harvested and iodixanol was removed by ultrafiltration (Amicon Ultra-4 Centrifugal Filter Unit (50 kDa cut-off, Millipore).

The genomic titers of DNAse resistant recombinant AAV6 particles were determined by quantitative PCR using the SYBR Green qPCR Master MIX 2 (ThermoScientific) and an ABI $7900 \mathrm{HT}$ cycler (ABI). Viral vectors were quantified using $\mathrm{T} 7 / \mathrm{SV} 40$ specific primers $\left(5^{\prime}\right.$-cctatagtgagtcgtattacgcgc and $5^{\prime}$ gctgcaataaacaagttgggccat).

Real-time PCR was done in $10 \mu \mathrm{l}$ with $0.3 \mu \mathrm{M}$ for each primer. Fluorescence was measured at the end of each annealing phase. AAV transfer plasmid was employed as a copy number standard. A standard curve for quantification was generated by serial dilutions of the respective vector plasmid DNA. The cycling conditions were as follows: $50^{\circ} \mathrm{C}$ for $2 \mathrm{~min}, 95^{\circ} \mathrm{C}$ for $10 \mathrm{~min}$, followed by $35 \mathrm{cycles}$ of $95^{\circ} \mathrm{C}$ for $15 \mathrm{sec}$ and $60^{\circ} \mathrm{C}$ for $60 \mathrm{sec}$. Calculations were done using SDS 2.4 software (ABI).

\section{Virus handling}

AAV-9: syn-DTA-GFP (AAV:syn-DTA) at a titre of $3.87 \times 10^{13} \mathrm{VP} \cdot \mathrm{ml}^{-1}$ was aliquoted and stored at $-80^{\circ} \mathrm{C}$. On the day of injection, viruses were removed and held at $4^{\circ} \mathrm{C}$, loaded into graduated glass pipettes (Drummond Scientific Company, Broomall, PA, USA), and placed into an electrode holder for pressure injection. The AAV:syn-DTA used the synapsin promoter, transducing neurons with higher tropism for the AAV 2/9 subtype, it did not transduce non-neuronal cells.

\section{Viral transduction of preBötC neurons}

Adult male Sprague Dawley rats (320-560 g) were anesthetized via intramuscular injection with ketamine (100 mg $\mathrm{kg}^{-1}$; Covetrus, Dumfries, UK) and medetomidine ( $250 \mu \mathrm{g} \cdot \mathrm{kg}^{-1}$; Covetrus, Dumfries, UK). Adequate anaesthesia was maintained with $0.5-2 \%$ Isofluorane (primal health care, India) in pure oxygen $\left(1 \mathrm{~L} \cdot \mathrm{min}^{-1}\right)$ throughout the surgery as need. Rats received a presurgical subcutaneous injection of atropine (120 $\mathrm{gg} \mathrm{kg}^{-1}$; Westward Pharmaceutical Co., Eatontown, NJ, USA) and meloxicam (2 mg $\mathrm{kg}^{-}$ 1; Norbrook Inc., Lenexa, KS, USA). Rats were placed in a prone position into a digital stereotaxic apparatus (Kopf Instruments, Tujunga, CA, USA) on a heating pad (TCAT 2-LV: Physitemp, Clifton, NJ, USA) and body temperature was maintained at a minimum of $33^{\circ} \mathrm{C}$ via a thermocouple. The head was angled so that the nose bar was $-18 \mathrm{~mm}$ below the intra-aural line. The injection arm was angled at $23^{\circ}$. Graduated glass pipettes containing the virus were placed stereotaxically into the preBötC (Fig $8 \mathrm{~A})$. The preBötC was defined as the area ventral to the semi-compact nucleus ambiguous (coordinates: $\pm 1.95 \mathrm{~mm}$ lateral and $-0.2 \mathrm{~mm}$ rostral and $+0.4 \mathrm{~mm}$ caudal, and $-2.95 \mathrm{~mm}$ ventral from the Obex; Fig 8 A). The virus solution was pressure injected ( $250-350 \mathrm{~nL}$ per side) bilaterally into all 4 sites. Pipettes were left in place for 3-5 minutes to prevent back flow of the virus solution up the pipette track. Postoperatively, rats received sub-cutaneous injections of buprenorphine $\left(100 \mu \mathrm{g} \cdot \mathrm{kg}^{-1}\right.$; Reckitt Benckiser, Slough, UK) and atipamezole (1 mg.kg-1). For sham surgeries pipettes were lowered $2 \mathrm{~mm}$ dorsoventral from the obex, with the same mediolateral and rostral co-ordinates. Rats were allowed 2 weeks for recovery and viral expression, with food and water ad libitum.

For preliminary experiments to determine the injection number and volume rats were injected with $\sim 300 \mathrm{~nL}$ of AAV:syn-DTA either at unilaterally at the aforementioned rostrocaudal coordinates or bilaterally in the aforementioned rostral coordinates. 


\section{EEG and EMG placement}

Following viral injections the head was levelled so the nose bar was set to 0 at the intraural line. Bur holes were drilled at 4 locations: 1 ) rostral to the coronal suture in mediolateral position central to the frontal bone plate that on the left of the sagittal suture to the most lateral extent of the bone; 2 ) In a rostrocaudal position central to the coronal and lamboidal suture in a position on the left of the sagittal suture to the most lateral extent of the parietal bone; 3) caudal to the coronal suture in a position on the right of the sagittal suture to the most lateral extent of the parietal bone, and; 4) rostral to the lamboidal suture in a position on the right of the sagittal suture to the most lateral extent of the parietal bone (Fig 8b). EMGS were placed bilaterally into the trapezius muscles. Electrodes were connected to custom built headmounts. Headmounts were adhered to the skull with superbond dental cement (prestige dental, Bedworth, UK), with additional support from Vertex Orthoplast coldcuring orthodontic acrylic resin (prestige dental, Bedworth, UK)

\section{Plethysmography}

Rats were placed into a custom-made $4.5 \mathrm{~L}$ plethysmography chamber (Fig $8 \mathrm{C}$ ), with an airflow rate of $2 \mathrm{~L} \cdot \mathrm{min}^{-1}$. Pressure transducer signals were amplified and filtered using the NeuroLog system (Digitimer, Welwyn Garden City, UK) connected to a 1401 interface. All data were acquired on a computer using Spike2 software (Cambridge Electronic Design, Cambridge, UK). In humans sleepdisordered breathing is defined as perturbed breathing for $\geq 10$ secs, which is $\sim 2$ breaths. In our experiments rats respiratory rate was 105 breaths $\min ^{-1}$, or 2 breaths every 1.15 seconds. For our analysis apnoeas were defined as an absence of breathing for $\geq 1.3$ seconds, and hypopnoeas by a drop in minute ventilation of $\geq 50 \%$ over the same time period. Apnoeas and hypopneas were scored by hand, and the length of each event recorded. Respiratory parameters were measured during quiet wakefulness at the beginning of the recording. Airflow measurements were used to calculate: tidal volume $\left(\mathrm{V}_{\mathrm{T}}\right)$, signal trough at the end of expiration subtracted from the peak signal during inspiration, converted to $\mathrm{mL}$ following calibration, and standardised to body weight); and respiratory frequency (f) calculated as breaths per minute using peak of inspiration to peak of inspiration averaged over 1 minute. Minute ventilation $\left(\mathrm{V}_{\mathrm{E}}\right)$ was calculated as $\mathrm{VT} \times f$.

\section{Sleep-Wake recordings}

Delta waves and neocortical EEG activity were obtained by differential recordings from electrodes 3 and 4 (Fig. 7B). Theta rhythms were obtained by differential recordings from electrodes 1 and 2 positioned in the dorsal hippocampus and the frontal cortex respectively (Fig. 7B). EEGs were bandpass filtered $0.5 \mathrm{~Hz}-60 \mathrm{~Hz}$. EMGs were recorded by differential recordings through the trapezoid wires (Fig. 7B) and bandpass filtered at $70 \mathrm{~Hz}-560 \mathrm{~Hz}$. Videos were recorded to aid with sleep-wake scoring. All data were acquired on a computer using Spike2 software (Cambridge Electronic Design, Cambridge, UK). EEG and EMG signals were processed using the OSD4 script: EEGs were band filtered with a power spectra of 2-4.5 Hz (delta) and 4.5-9 Hz (theta). EMGs were smoothed over 1 second. The 3 hour long recordings were separated in 5 second epochs. Sleep-wake scoring epochs were categorised into 4 predefine classes:1) WAKE - low amplitude desynchronized EEG activity and high EEG power; 2) NREM - high amplitude EEG delta waves and low EMG power; 3) REM - Continuous low amplitude EEG theta activity leading to high theta:delta (T:D) ratio, and no EMG power, muscular paralysis could be observed on the associated video; 4) DOUBT - Epochs that could not be defined into the previous 3 categories.

\section{Barnes Maze}

The Barnes maze is a $122 \mathrm{~cm}$ diameter circular maze with $20 \times 90 \mathrm{~cm}$ holes; 19 false holes and one escape hole (Stoelting, Dublin, Ireland) (Fig 8E). The maze is one metre off the ground. Spatial cues of different colour and shape are spaced evenly around the maze at regular intervals in plain sight of the rat. The surface of the table is brightly lit ( $>1500$ lux) to create an adverse environment. Rats underwent a 17 day Barnes maze protocol: Days 1-3 (learning phase) the escape hole (randomly assigned on day 1) contained an incentive (peanut butter); days 4-12 (acquisition phase) the incentive is provided in the home cage after the test, allowing the rat to be rewarded for completing the test 
but removing the olfactory stimulus from the maze; days 13-17 (reversal phase) the escape hole is placed at $180^{\circ}$, this phase is to test the ability of the rats to unlearn a task. The task was completed when the mouse entered the exit box. All runs were recorded using a camera system (Henelec Model 335 BWL SONY) attached to a computer for offline analysis (Any-MAZE v4.96, Stoelting). Total distance, duration, and exit errors were measured. Exit errors was measured by calculating the number of times the rat visit the exit hole but remained on the maze. Heat maps of the rats movements were created for determination of the search strategy. These were: random: no consistent pattern, $>2$ crossings of the open field; serial: a hole-by-hole progression with $\geq 3$ consecutive holes visited; and spatial: moving directly to the exit hole \pm 2 holes and no deviation outside of the quadrant ${ }^{70}$.

\section{Y Maze (forced alternation)}

The spontaneous spatial novelty preference test was conducted using a radial arm maze (Stoelting, Dublin, Ireland), adapted into a Y-maze. Each arm was $50 \mathrm{~cm}$ long, $10 \mathrm{~cm}$ wide, with $13-\mathrm{cm}$-high walls (Fig 8D). All rats were placed into the same entry arm. For the first trial, one of the arms of the Y-maze was blocked, therefore rats could either go left or right according to a pseudorandom sequence. Rats could also can move in the central region. First trial lasted for 10 mins. After 1 hour, rats underwent a second trial to assess short term memory. The test was repeated with access to all arms. All runs were recorded using a camera system (Henelec Model 335 BWL SONY) attached to a computer for offline analysis (Any-MAZE v4.96, Stoelting). Total distance, duration, and number of entries into each arm were measured. An entry into an arm was defined as placement of 2 paws into that arm.

\section{Immunology}

Rats were anesthetized with Isofluorane (0.5-2\%; primal health care, India) in room air (1 L. $\left.\mathrm{min}^{-1}\right)$. Inferior vena cava blood samples were taken and placed on ice before being spun at $13 \mathrm{~K} \mathrm{RPM}$ at $4^{\circ} \mathrm{C}$ for 10 mins and the plasma collected; samples were stored at $-80^{\circ} \mathrm{C}$. Plasma sample were run on sandwich ELISA kits testing 8 inflammatory cytokines (TNF- $\alpha$, IL-6, MCP-1, IFN- $\gamma$, Rantes, MIP, IL-1a, IL1b; Signosis EA-1201) and noradrenaline (whole antibody; Cusabio CSB-E07021h). Optical densities were converted to concentrations via standard curves (Signosis EA-1202). Due to the variability in the ELISA kits, measurements that did not lie on the linear part of the curve were discarded and the samples were run again. To further reduce the impact of the variability of the ELSIA kits, samples were pseudorandomised to kits and run in duplicate on different kits.

\section{Collection of brain tissue}

Rats were humanely killed by overdose of Isofluorane. The brains were removed. The medulla and 1 cortical hemisphere were placed into $4 \%$ paraformaldehyde (PFA) for immunocyctochemistry. The other cortical hemisphere was placed into ice cold saline for electrophysiological studies.

\section{Immunocytochemistry}

The brain was postfixed overnight in PFA $\left(4^{\circ} \mathrm{C}\right)$, and cryoprotected in $30 \%$ sucrose. Brainstems and hippocampi were serially sectioned at $40 \mu \mathrm{m}$. Free-floating sections were placed in blocking solution (phosphate buffered saline [PBS] $+5 \%$ bovine serum albumin $+0.1 \%$ Triton X-100) and incubated for 1 hour. Slices were further incubated overnight in blocking solution and primary antibodies: Medulla Rabbit anti-substance P receptor - (1:500, Merck Millipore ab5060) and mouse anti-NeuN (1:100, Merck Millipore MAB337) antibody; Hippocampus - Goat anti-lba-1 (1:83; Abcam, ab5076) antibody. Slices were washed in blocking solution ( $6 \times 5 \mathrm{mins})$ and then incubated for 2-4 hrs in blocking solution containing secondary antibodies: Medulla - donkey anti-rabbit Alexa Fluor 568 (1:250; Abcam ab150074), or donkey anti-mouse Alexa Fluor 568 (1:250; Invitrogen A10037) antibody; Hippocampus - donkey anti-goat Alexa Fluor 488 (1:250; Invitrogen, A11055). Slices were mounted, coverslipped (Medulla: Cytoseal 60 [Electron Microscopy Sciences, Hatfield, PA], Hippocampus: Vectashield Antifade Mounting Medium with DAPI [Vectorlabs, Burlingame]).

Slices were examined using a Zeiss 880 confocal fluorescent microscope with Zen black and Zen blue software. For the medulla: To determine the efficacy of viral transduction, cell counts of NeuN positive 
cells were performed in representative $40 \mu \mathrm{m}$ sections throughout transduced brainstems, within a $600 \mu \mathrm{m}$ diameter circle below the semi-compact nucleus ambiguous ${ }^{24}$. To establish the loss of rhythm generating preBotC neurons, fluorescence intensity of NK1R staining was assessed in ImageJ within the same circle. The numbers of neurons and NK1R intensity for each animal are provided in the text as means \pm SD. For the hippocampus: To identify activated microglia, we counted DAPI stained nuclei that we encapsulated with Iba1, anywhere in the visual field. Cell counts provided in the text are reported as means \pm SD.

\section{Extracellular recording of fEPSPs in hippocampal slices} rigs to increase productivity. The data from the two rigs was not significantly different and so was pooled.

Recording on Rig 1: A slice was transferred to the recording chamber submerged in aCSF (124 mM $\mathrm{NaCl}, 3 \mathrm{mM} \mathrm{KCl}, 2 \mathrm{mM} \mathrm{CaCl}, 26 \mathrm{mM} \mathrm{NaHCO}_{3}, 1.25 \mathrm{mM} \mathrm{NaH}_{2} \mathrm{PO}_{4}, 1 \mathrm{mM} \mathrm{MgSO}$, $10 \mathrm{mM}$ d-glucose saturated with $95 \% \mathrm{O} 2-5 \% \mathrm{CO} 2, \mathrm{pH} 7.5)$ and perfused at $4-6 \mathrm{ml} / \mathrm{min}\left(32^{\circ} \mathrm{C}\right)$. The slice was placed on a grid allowing perfusion above and below the tissue and all tubing (Tygon) was gas tight (to prevent loss of oxygen). To record field excitatory postsynaptic potentials (fEPSPs), an aCSF-filled microelectrode was placed on the surface of stratum radiatum in CA1 (Fig 8F). A bipolar concentric stimulating electrode (FHC) controlled by an isolated pulse stimulator model 2100 (AM Systems, WA) was used to evoke fEPSPs at the Schaffer collateral-commissural pathway (Fig 8F). Field (f)EPSPs were evoked every $30 \mathrm{~s}(0.03 \mathrm{~Hz})$. Stimulus input/output curves for fEPSPs were generated using stimulus strength of 1-5 $\mathrm{V}$ for all slices (stimuli duration $0.2 \mathrm{~ms}$ ). The paired-pulse ratio was measured over an interval range of 20 to $500 \mathrm{~ms}$ (ratio averaged for 5 repeats at each interval). For the synaptic plasticity experiments, the stimulus strength was set to produce a fEPSP slope $\sim 40 \%$ of the maximum response. Long-term potentiation (LTP) was induced by theta burst stimulation (TBS, 3 trains separated by 20s with train consisting of 10 bursts of 4 pulses at $100 \mathrm{~Hz}$, separated by $200 \mathrm{~ms}$ ). Signals were filtered at $3 \mathrm{kHz}$ and digitised on-line (10 kHz) with a Micro CED (Mark 2) interface controlled by Spike software (Vs 6.1, Cambridge Electronic Design, Cambridge UK). The fEPSP slope was measured from a $1 \mathrm{~ms}$ linear region following the fibre volley. All I/O curves and PPF comparisons were made from this recording set up

468 Recording on rig 2: The slice was placed on a grid allowing perfusion $\left(6-7 \mathrm{ml} / \mathrm{min}\right.$ at $\left.32^{\circ} \mathrm{C}\right)$ above and 469 below the tissue and all tubing (Tygon) was gas tight (to prevent loss of oxygen) Field excitatory postsynaptic potentials were recorded as outlined for rig 1. A bipolar concentric stimulating electrode (FHC) controlled by a DS3 isolated current stimulator (Digitimer, UK) with field (f)EPSPs evoked every $45 \mathrm{~s}$. Stimulus input/output curves for fEPSPs were generated using stimulus strength of 20-300 $\mu \mathrm{A}$ (stimuli duration $0.2 \mathrm{~ms}$ ), stimulus strength was set to produce a fEPSP slope $\sim 40 \%$ of the maximum response. Long-term potentiation was induced by theta burst stimulation (as above for rig 1). Signals were acquired using WinLTP software (Vs 2.3, WinLTP Ltd, Bristol, UK).

\section{Experimental paradigm}

For the transduction, the order of rats and whether they received viral injections or were shams were pseudorandomised. Experimenters were blinded to the condition of the rats. Rats were acclimated to the plethysmography chamber the $2^{\text {nd }}$ week post-surgery. Plethysmography began 3 weeks post transduction and continued for 6 weeks. Rats then underwent 1 day of Y-maze, and 17 days of Barnes maze, testing the order of which was randomised for each batch. Rats were then allowed 1 more week before tissue collection and LTP experiments were performed. Experimenters were unblinded to the condition of the animals once analysis had been performed (fig 8G).

484 Preliminary experiments to determine the injection volume required to induce sleep apnoea were 
once analysis had been performed. Given the lack of sleep apnoea induced in rats in the preliminary experiments, dual injected rats were pooled into he sham operated rats for analysis.

\section{Data analysis}

Outliers were removed after Iglewicz and Hoaglin's robust test for multiple outliers with an outlier criterion: Modified Z score $\geq 3.5$ (https://contchart.com/outliers.aspx). Sharipo-Wilk tests were performed on sham operated groups.

Data for preBotC cell counts $(p=0.5)$, preBotC NK1R intensity $(p=0.1)$, AHI $(p=0.3)$, time spent in disordered breathing $(p=0.25), V_{T}(p=0.3), f(p=0.1), V_{E}(p=0.2)$, Wake $(p=0.2)$, NREM $(p=0.1)$, and REM $(p=0.4)$, IBA1 counts $(0.6)$, IFNy $(p=0.06)$, IL- $1 a(P=0.96), I L-1 b(P=0.4)$, and IL-6 $(P=0.3)$, CRP (0.9), NA ( $P=0.07)$ were deemed Gaussian by a Sharipo-Wilk test for normality, and tested an unpaired T-Test. Data are expressed as mean \pm SD.

Data for $Y$-maze novel arm entries $(P=0.6), Y$-maze novel duration $(P=0.98), Y$-maze novel arm distance $(P=0.06)$, and paired pulse facilitation $(P=0.6)$ were deemed Gaussian by a Sharipo-Wilk test for normality, and tested via a repeated measures 2 way ANOVA with a Bonferroni correction. Data are expressed as mean \pm SD.

Data for CA1 IBA1 counts $(p=0.02)$,TNFa $(p=0.02)$, MCP-1 $(p=0.03)$, MIP $(0.01)$, and Rantes $(0.02)$, were deemed non-Gaussian by a Sharipo-Wilk test for normality, and tested via a Kruskal-Wallis Test. $68 \%$ of data are contained within $1 \mathrm{SD}$, to make data comparable we calculated the data range from $15^{\text {th }}$ to the $85^{\text {th }}$ quartile, herein known as Range ${ }_{70}$. Data are expressed as median \pm Range $_{70}$, and displayed graphically as median \pm quartile 15-85.

Data for Barnes duration $(P=0.0)$, Barnes maze distance $(P=0.0)$, Barnes maze Escape hole failures $(P=0.0)$, and LTP $(P=0.0002)$ were deemed non-Gaussian by a Sharipo-Wilk test for normality, and tested via a repeated measures 2 way ANOVA with a Sidak correction. Data are expressed as mean \pm SEM.

Data for input-output curves $(\mathrm{P}=0.00005)$, were deemed non-Gaussian by a Sharipo-Wilk test for normality, and tested via a 2 way ANOVA with a Sidak correction. Data are expressed as mean \pm SEM.

\section{References}

1 Tan, A. et al. Prevalence of sleep-disordered breathing in a multiethnic Asian population in Singapore: A community-based study. Respirology 21, 943-950, doi:10.1111/resp.12747 (2016).

2 Heinzer, R. et al. Prevalence of sleep-disordered breathing in the general population: the HypnoLaus study. Lancet Respir Med 3, 310-318, doi:10.1016/S2213-2600(15)00043-0 (2015).

3 Rowley, J. A., Zahn, B. R., Babcock, M. A. \& Badr, M. S. The effect of rapid eye movement (REM) sleep on upper airway mechanics in normal human subjects. J Physiol 510 ( Pt 3), 963-976, doi:10.1111/j.1469-7793.1998.00963.x (1998).

4 Xie, A. Effect of sleep on breathing - Why recurrent apneas are only seen during sleep. $J$ Thorac Dis 4, 194-197, doi:10.3978/j.issn.2072-1439.2011.04.04 (2012).

5 Remmers, J. E., deGroot, W. J., Sauerland, E. K. \& Anch, A. M. Pathogenesis of upper airway occlusion during sleep. J Appl Physiol Respir Environ Exerc Physiol 44, 931-938, doi:10.1152/jappl.1978.44.6.931 (1978).

6 Gupta, R., Das, S., Shankar Shilpi, U., Sindhwani, G. \& Khandoori, R. Idiopathic central sleep apnea during REM sleep. Somnologie 20, 150-154, doi:10.1007/s11818-016-0050-z (2016).

7 Loadsman, J. A. \& Wilcox, I. Is obstructive sleep apnoea a rapid eye movement-predominant phenomenon? BJA: British Journal of Anaesthesia 85, 354-358, doi:10.1093/bja/85.3.354 (2000).

8 Veasey, S. C. \& Rosen, I. M. Obstructive Sleep Apnea in Adults. N Engl J Med 380, 1442-1449, doi:10.1056/NEJMcp1816152 (2019).

9 Maniaci, A. et al. Oxidative Stress and Inflammation Biomarker Expression in Obstructive Sleep Apnea Patients. J Clin Med 10, doi:10.3390/jcm10020277 (2021). 
10 McNicholas, W. T. Obstructive sleep apnea and inflammation. Prog Cardiovasc Dis 51, 392-399, doi:10.1016/j.pcad.2008.10.005 (2009).

11 Gottlieb, D. J. et al. Prospective study of obstructive sleep apnea and incident coronary heart disease and heart failure: the sleep heart health study. Circulation 122, 352-360, doi:10.1161/CIRCULATIONAHA.109.901801 (2010).

12 Reutrakul, S. \& Mokhlesi, B. Obstructive Sleep Apnea and Diabetes: A State of the Art Review. Chest 152, 1070-1086, doi:10.1016/j.chest.2017.05.009 (2017).

13 Bahia, C. M. C. d. S. \& Pereira, J. S. Obstructive sleep apnea and neurodegenerative diseases: A bidirectional relation. Dement Neuropsychol 9, 9-15, doi:10.1590/S1980-57642015DN91000003 (2015).

14 Matuska, P., Kara, T., Homolka, P. \& Belehrad, M. Advances in the management of sleep-disordered breathing in heart failure. Cor et Vasa 55, e411-e418, doi:http://dx.doi.org/10.1016/i.crvasa.2013.06.005 (2013).

15 Lanfranchi, P. A. et al. Prognostic Value of Nocturnal Cheyne-Stokes Respiration in Chronic Heart Failure. Circulation 99, 1435 (1999).

16 Javaheri, S., Caref, E. B., Chen, E., Tong, K. B. \& Abraham, W. T. Sleep apnea testing and outcomes in a large cohort of medicare beneficiaries with newly diagnosed heart failure. Am J Respir Crit Care Med 183, 539-546, doi:10.1164/rccm.201003-04060C (2011).

17 Lavergne, F. et al. Atrial fibrillation and sleep-disordered breathing. J Thorac Dis 7, E575-E584 (2015).

18 Zuliani, G. et al. Markers of endothelial dysfunction in older subjects with late onset Alzheimer's disease or vascular dementia. J Neurol Sci 272, 164-170, doi:10.1016/j.jns.2008.05.020 (2008).

19 Calbet, J. A. L. Chronic hypoxia increases blood pressure and noradrenaline spillover in healthy humans. J Physiol 551, 379-386, doi:10.1113/jphysiol.2003.045112 (2003).

20 Drager, L. F., Polotsky, V. Y. \& Lorenzi-Filho, G. Obstructive sleep apnea: an emerging risk factor for atherosclerosis. Chest 140, 534-542, doi:10.1378/chest.10-2223 (2011).

21 Brown, D. L. et al. Sleep-Disordered Breathing Is Associated With Recurrent Ischemic Stroke. Stroke 50, 571-576, doi:10.1161/STROKEAHA.118.023807 (2019).

22 Dusak, A. et al. Correlation between hippocampal volume and excessive daytime sleepiness in obstructive sleep apnea syndrome. Eur Rev Med Pharmacol Sci 17, 1198-1204 (2013).

23 Yang, Q., Wang, Y., Feng, J., Cao, J. \& Chen, B. Intermittent hypoxia from obstructive sleep apnea may cause neuronal impairment and dysfunction in central nervous system: the potential roles played by microglia. Neuropsychiatr Dis Treat 9, 1077-1086, doi:10.2147/NDT.S49868 (2013).

24 McKay, L. C. \& Feldman, J. L. Unilateral ablation of preBotzinger Complex disrupts breathing during sleep but not wakefulness. Am. J. Respir. Crit. Care Med. 178, 89-95, doi:10.1164/rccm.200712$19010 C$ (2008).

25 Ziegler, M. G. et al. Sleep apnea, norepinephrine-release rate, and daytime hypertension. Sleep 20, 224-231, doi:10.1093/sleep/20.3.224 (1997).

26 Monif, M. et al. Interleukin-1 $\beta$ has trophic effects in microglia and its release is mediated by P2X7R pore. Journal of Neuroinflammation 13, 173, doi:10.1186/s12974-016-0621-8 (2016).

27 Basu, A., Krady, J. K. \& Levison, S. W. Interleukin-1: a master regulator of neuroinflammation. J Neurosci Res 78, 151-156, doi:10.1002/jnr.20266 (2004).

$28 \mathrm{Ma}$, J. et al. Chronic brain inflammation causes a reduction in GluN2A and GluN2B subunits of NMDA receptors and an increase in the phosphorylation of mitogen-activated protein kinases in the hippocampus. Molecular Brain 7, 33, doi:10.1186/1756-6606-7-33 (2014).

29 Strauss, N. \& Hendee, E. D. The effect of diphtheria toxin on the metabolism of HeLa cells. J Exp Med 109, 145-163, doi:10.1084/jem.109.2.145 (1959).

30 Zalman, L. S. \& Wisnieski, B. J. Mechanism of insertion of diphtheria toxin: peptide entry and pore size determinations. Proc Natl Acad Sci U S A 81, 3341-3345, doi:10.1073/pnas.81.11.3341 (1984). 
31 Zaremba, S. et al. Continuous Positive Airway Pressure Mitigates Opioid-induced Worsening of Sleep-disordered Breathing Early after Bariatric Surgery. Anesthesiology 125, 92-104, doi:10.1097/ALN.0000000000001160 (2016).

32 Aldrich, M., Eiser, A., Lee, M. \& Shipley, J. E. Effects of continuous positive airway pressure on phasic events of REM sleep in patients with obstructive sleep apnea. Sleep 12, 413-419, doi:10.1093/sleep/12.5.413 (1989).

33 Moe, K. E., Vitiello, M. V., Larsen, L. H. \& Prinz, P. N. Sleep/wake patterns In Alzheimer's disease: relationships with cognition and function. J Sleep Res 4, 15-20, doi:10.1111/j.13652869.1995.tb00145.x (1995).

34 Holth, J. K., Patel, T. K. \& Holtzman, D. M. Sleep in Alzheimer's Disease-Beyond Amyloid. Neurobiol Sleep Circ Rhythms 2, 4-14, doi:https://doi.org/10.1016/j.nbscr.2016.08.002 (2017).

35 Boyce, R., Glasgow, S. D., Williams, S. \& Adamantidis, A. Causal evidence for the role of REM sleep theta rhythm in contextual memory consolidation. Science 352, 812, doi:10.1126/science.aad5252 (2016).

36 Ning, Y. et al. IFNgamma restores breast cancer sensitivity to fulvestrant by regulating STAT1, IFN regulatory factor 1, NF-kappaB, BCL2 family members, and signaling to caspase-dependent apoptosis. Mol Cancer Ther 9, 1274-1285, doi:10.1158/1535-7163.MCT-09-1169 (2010).

37 Castro, F., Cardoso, A. P., Gonçalves, R. M., Serre, K. \& Oliveira, M. J. Interferon-Gamma at the Crossroads of Tumor Immune Surveillance or Evasion. Front Immunol 9, doi:10.3389/fimmu.2018.00847 (2018).

38 Martinez, F. O., Gordon, S., Locati, M. \& Mantovani, A. Transcriptional Profiling of the Human Monocyte-to-Macrophage Differentiation and Polarization: New Molecules and Patterns of Gene Expression. The Journal of Immunology 177, 7303, doi:10.4049/jimmunol.177.10.7303 (2006).

39 Tsuchiya, K. et al. Gasdermin D mediates the maturation and release of IL-1 $\alpha$ downstream of inflammasomes. Cell Rep 34, 108887, doi:https://doi.org/10.1016/j.celrep.2021.108887 (2021).

40 Rock, K. L., Latz, E., Ontiveros, F. \& Kono, H. The sterile inflammatory response. Annu Rev Immunol 28, 321-342, doi:10.1146/annurev-immunol-030409-101311 (2010).

41 Martin, C. A. \& Dorf, M. E. Differential regulation of interleukin-6, macrophage inflammatory protein-1, and JE/MCP-1 cytokine expression in macrophage cell lines. Cell Immunol 135, 245-258, doi:10.1016/0008-8749(91)90269-h (1991).

42 Liang, D.-Y. et al. Caspase-1 modulates incisional sensitization and inflammation. Anesthesiology 113, 945-956, doi:10.1097/ALN.0b013e3181ee2f17 (2010).

43 Marfaing-Koka, A. et al. Regulation of the production of the RANTES chemokine by endothelial cells. Synergistic induction by IFN-gamma plus TNF-alpha and inhibition by IL-4 and IL-13. The J Immunol 154, 1870-1878 (1995).

44 Yamana, J., Santos, L. \& Morand, E. Enhanced induction of LPS-induced fibroblast MCP-1 by interferon- $\gamma$ : Involvement of JNK and MAPK phosphatase-1. Cell Immunol 255, 26-32, doi:https://doi.org/10.1016/j.cellimm.2008.09.003 (2009).

45 Castell, J. V. et al. Interleukin-6 is the major regulator of acute phase protein synthesis in adult human hepatocytes. FEBS Letters 242, 237-239, doi:https://doi.org/10.1016/0014-5793(89)804764 (1989).

46 Vila-del Sol, V., Punzón, C. \& Fresno, M. IFN-gamma-induced TNF-alpha expression is regulated by interferon regulatory factors 1 and 8 in mouse macrophages. J Immunol 181, 4461-4470, doi:10.4049/jimmunol.181.7.4461 (2008).

47 Wang, N. et al. Elevated CO2 selectively inhibits interleukin-6 and tumor necrosis factor expression and decreases phagocytosis in the macrophage. Faseb j 24, 2178-2190, doi:10.1096/fj.09-136895 (2010).

48 Guttmann, R. P. \& Johnson, G. V. Oxidative stress inhibits calpain activity in situ. J Biol Chem 273, 13331-13338, doi:10.1074/jbc.273.21.13331 (1998).

$49 \mathrm{Kim}, \mathrm{N}$. G. et al. Hypoxic induction of caspase-11/caspase-1/interleukin-1beta in brain microglia. Brain Res Mol Brain Res 114, 107-114, doi:10.1016/s0169-328x(03)00135-9 (2003). 
50 Zhang, W. H. et al. Fundamental role of the Rip2/caspase-1 pathway in hypoxia and ischemiainduced neuronal cell death. Proc Natl Acad Sci 100, 16012-16017, doi:10.1073/pnas.2534856100 (2003).

51 Roman, J. et al. T-cell activation under hypoxic conditions enhances IFN-gamma secretion. Am J Respir Cell Mol Biol 42, 123-128, doi:10.1165/rcmb.2008-01390C (2010).

$52 \mathrm{Kim}, \mathrm{S}$. Y. et al. Hypoxic stress up-regulates the expression of Toll-like receptor 4 in macrophages via hypoxia-inducible factor. Immunology 129, 516-524, doi:10.1111/j.1365-2567.2009.03203.x (2010).

53 Cowell, R. M., Xu, H., Galasso, J. M. \& Silverstein, F. S. Hypoxic-Ischemic Injury Induces Macrophage Inflammatory Protein-1a Expression in Immature Rat Brain. Stroke 33, 795-801, doi:10.1161/hs0302.103740 (2002).

54 Dhupar, R. et al. Interferon Regulatory Factor 1 Mediates Acetylation and Release of High Mobility Group Box 1 from Hepatocytes During Murine Liver Ischemia-Reperfusion Injury. Shock 35 (2011).

$55 \mathrm{Kim}, \mathrm{J}$. H. et al. Epstein-Barr virus EBNA2 directs doxorubicin resistance of B cell lymphoma through CCL3 and CCL4-mediated activation of NF-KB and Btk. Oncotarget 8, 5361-5370, doi:10.18632/oncotarget.14243 (2017).

56 Laghlali, G., Lawlor, K. E. \& Tate, M. D. Die Another Way: Interplay between Influenza A Virus, Inflammation and Cell Death. Viruses 12, doi:10.3390/v12040401 (2020).

57 Abd-El-Basset, E. M., Rao, M. S. \& Alsaqobi, A. Interferon-Gamma and Interleukin-1Beta Enhance the Secretion of Brain-Derived Neurotrophic Factor and Promotes the Survival of Cortical Neurons in Brain Injury. Neurosci Insights 15, 2633105520947081, doi:10.1177/2633105520947081 (2020).

58 Papageorgiou, I. E. et al. TLR4-activated microglia require IFN- $\gamma$ to induce severe neuronal dysfunction and death in situ. Proc Natl Acad Sci 113, 212-217, doi:10.1073/pnas.1513853113 (2016).

59 Monteiro, S. et al. Absence of IFN $\gamma$ promotes hippocampal plasticity and enhances cognitive performance. Transll Psychiatry 6, e707-e707, doi:10.1038/tp.2015.194 (2016).

60 Torelli, F. et al. Cognitive profile and brain morphological changes in obstructive sleep apnea. Neuroimage 54, 787-793, doi:10.1016/j.neuroimage.2010.09.065 (2011).

61 Yoshiyama, Y. et al. Synapse loss and microglial activation precede tangles in a P301S tauopathy mouse model. Neuron 53, 337-351, doi:10.1016/j.neuron.2007.01.010 (2007).

62 Olsson, B. et al. Microglial Markers are Elevated in the Prodromal Phase of Alzheimer's Disease and Vascular Dementia. JAD 33, 45-53, doi:10.3233/JAD-2012-120787 (2013).

$63 \mathrm{Bu}, \mathrm{X}$. L. et al. Serum amyloid-beta levels are increased in patients with obstructive sleep apnea syndrome. Sci Rep 5, 13917, doi:10.1038/srep13917 (2015).

64 Pan, W. \& Kastin, A. J. Can sleep apnea cause Alzheimer's disease? Neurosci Biobehav Rev 47, 656669, doi:10.1016/j.neubiorev.2014.10.019 (2014).

65 Sharafkhaneh, A., Giray, N., Richardson, P., Young, T. \& Hirshkowitz, M. Association of Psychiatric Disorders and Sleep Apnea in a Large Cohort. Sleep 28, 1405-1411, doi:10.1093/sleep/28.11.1405 (2005).

66 Aloia, M. S., Arnedt, J. T., Davis, J. D., Riggs, R. L. \& Byrd, D. Neuropsychological sequelae of obstructive sleep apnea-hypopnea syndrome: a critical review. J Int Neuropsychol Soc 10, 772-785, doi:10.1017/s1355617704105134 (2004).

67 Saunamäki, T. \& Jehkonen, M. A review of executive functions in obstructive sleep apnea syndrome. Acta Neurol Scand 115, 1-11, doi:10.1111/j.1600-0404.2006.00744.x (2007).

68 Ooms, S. \& Ju, Y.-E. Treatment of Sleep Disorders in Dementia. Curr Treat Options Neurol 18, 4040, doi:10.1007/s11940-016-0424-3 (2016).

69 Borak, J., Cif, Koziej, M., Matuszewski, A. \& ZieliDski, J. Effects of CPAP treatment on psychological status in patients with severe obstructive sleep apnoea. J Sleep Res 5 (1996).

70 Wall, M. J. et al. The Temporal Dynamics of Arc Expression Regulate Cognitive Flexibility. Neuron 98, 1124-1132.e1127, doi:10.1016/j.neuron.2018.05.012 (2018). 
A

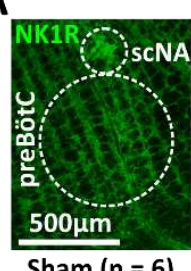

Sham $(n=6)$

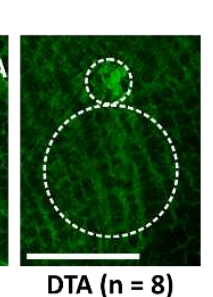

AWAKE

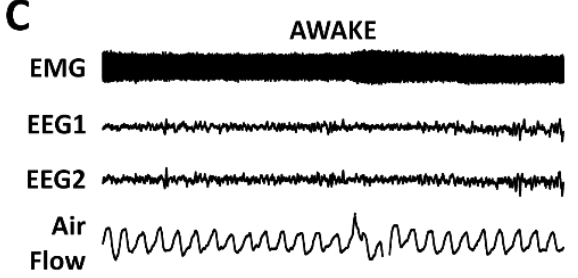

D

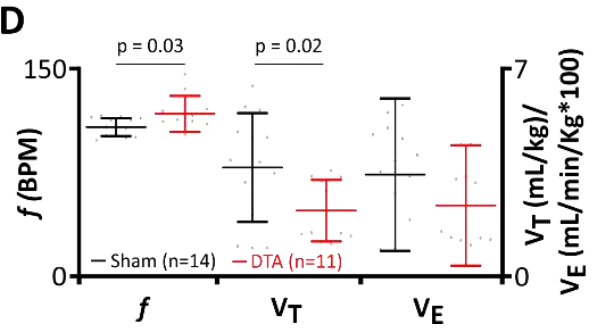

B

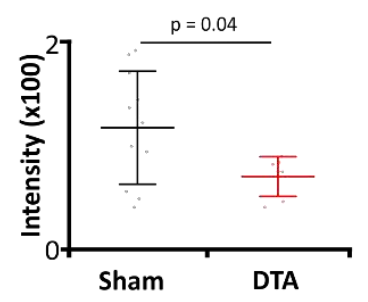

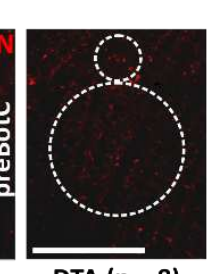

DTA $(n=8)$

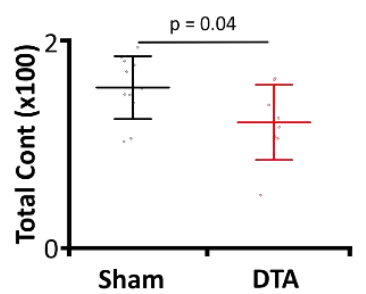

REM

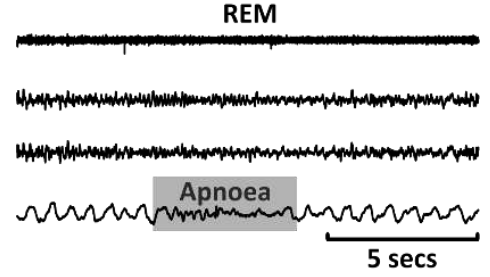

F
687

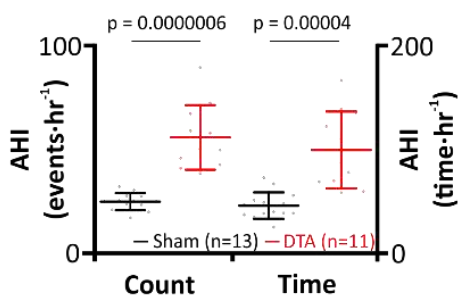

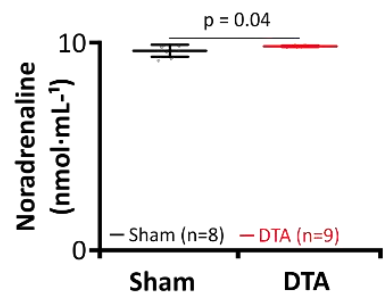

Fig. 1: preBötC lesions create a physiologically realistic experimental model of SA. A) Micrographs showing loss of NK1R immunoreactivity in the preBötC neurons following transduction with AAV:SynDTA (left) with group data (right). B) Micrographs showing loss of preBötC neurons following transduction with AAV:Syn-DTA (left) with group data (right). C) Plethysmograph traces from rats post DTA injection, showing respiratory movements as measured by changes in air flow in a plethysmograph, and sleep-wake state as measured by EEG and EMG electrodes. D) Respiratory parameters during wakefulness in DTA injected (red) and sham (black) rats. Left axis shows values for frequency $(f)$, right axis for tidal volume $\left(V_{T}\right)$ and minute ventilation $\left(V_{e}\right)$. E) Progression of SA after transduction with DTA (red) compared to shams (black). AHI - apnoea-hypopnoea index. F) preBötC lesions with DTA leads to systemic hypoxia. Noradrenaline $(\mathrm{ng} / \mathrm{mL})$ from sham $(\mathrm{n}=8)$ and DTA $(n=9)$ rats. Data represented as mean $\pm S D$, with individual data points. 


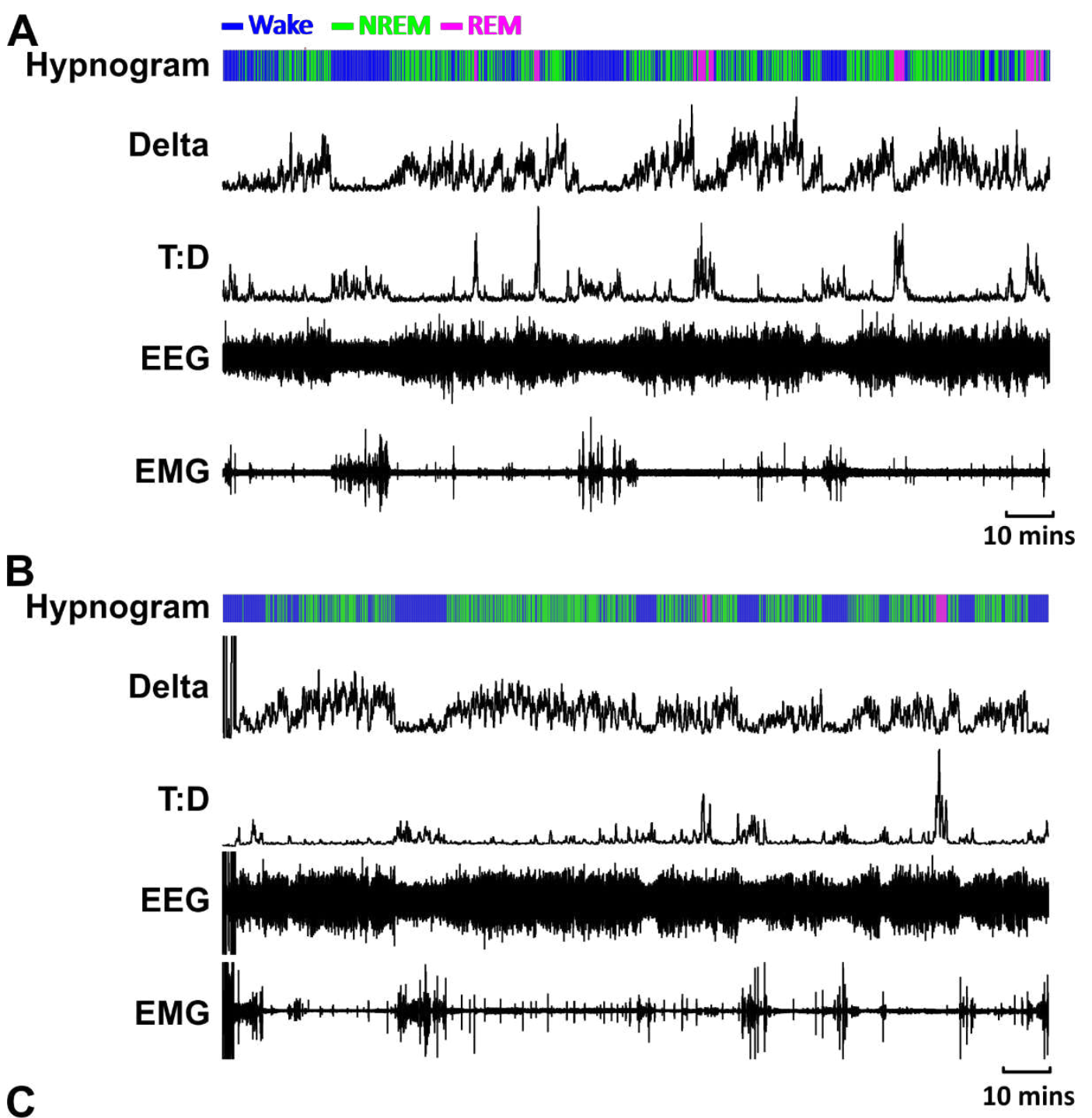

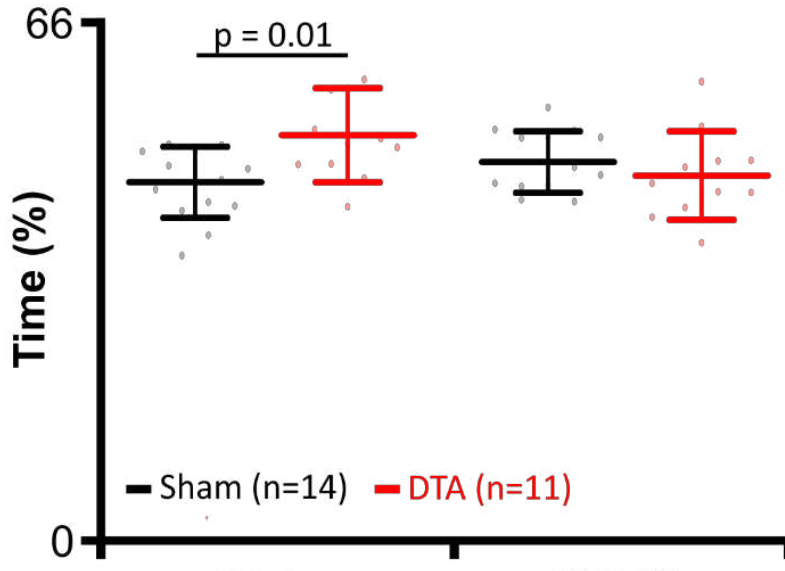

Wake

NREM

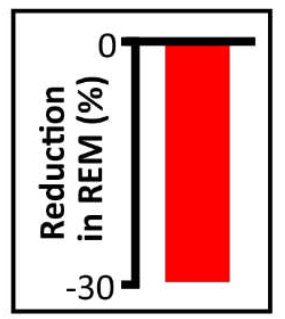

$p=0.003$

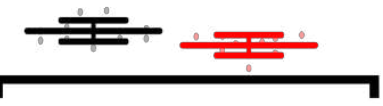

REM

Fig.2: Induction of sleep apnoea leads to a reduction in REM sleep. A) Data from an individual sham operated rat. B) Data from an individual DTA injected rat. A+B) EMG recordings were used to determine activity and to identify periods of REM and NREM sleep. EEG recordings were used to determine delta wave activity for NREM sleep and to calculate the theta-delta ratio (T:D) for REM sleep. Hypnograms show time spent awake and in REM and NREM sleep. C) DTA rats (red) spend less time in REM sleep (insert) than shams (black), with the lost REM time spent awake. Data represented as mean $\pm S D$, with individual data points. 

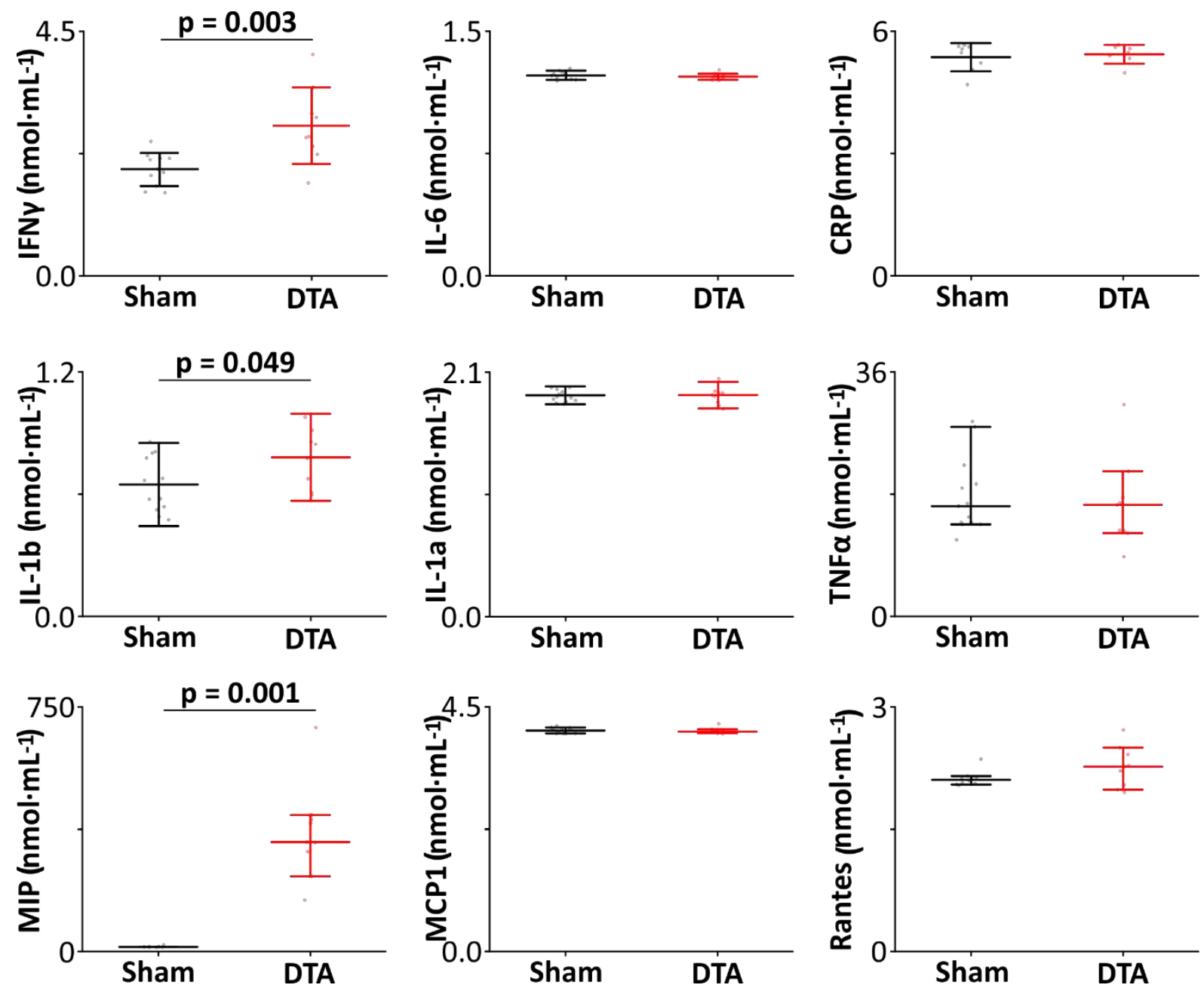

Fig. 3: Induction of sleep apnoea leads to a systemic inflammation. A) Inflammatory profiles $(\mathrm{nmol} / \mathrm{mL})$ from sham operated $(\mathrm{n}=14)$ and DTA-injected $(\mathrm{n}=9)$ rats with preBötC lesions. Data represented as median with $70 \%$ range (TNF $\alpha$, MCP-1, MIP, and RANTES), and as mean \pm SD (IFN $\gamma$, IL$1 a, I L-1 b, I L-6$, and CRP) with individual data points. Data represented as mean \pm SD, with individual data points. 

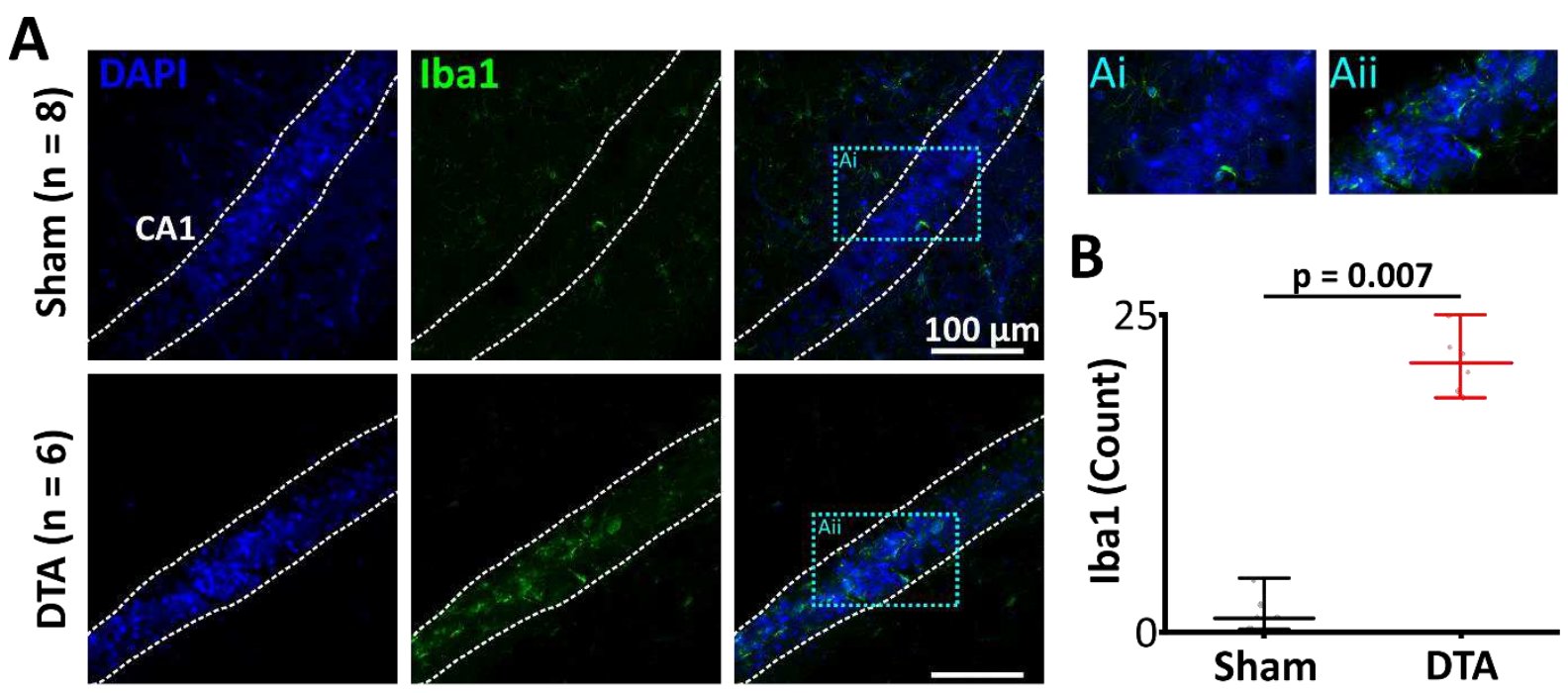

B
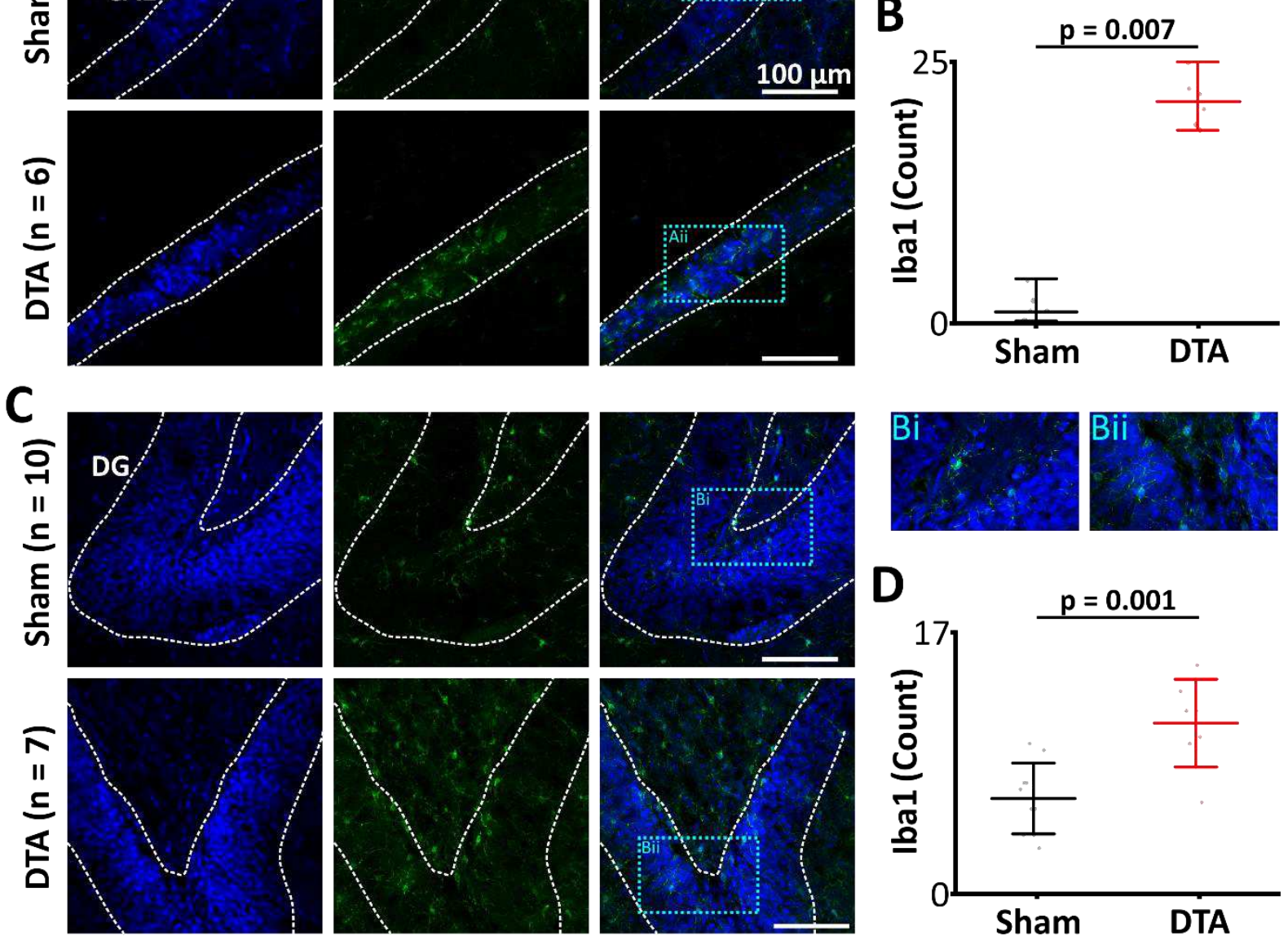

Sham

DTA

719 Fig. 4: Induction of sleep apnoea leads to a neural inflammation. $A+C)$ Micrographs showing activated microglia, a marker of neural inflammation, through Iba1 expression. Blue boxes represent expanded areas shown in $i$ and ii. Neural inflammation can be seen in the; A) CA1 region and C) dentate gyrus (DG). B+C) Group data are displayed in B) CA1 and D) DG. Data represented as mean \pm $\mathrm{SD}$, with individual data points 
A

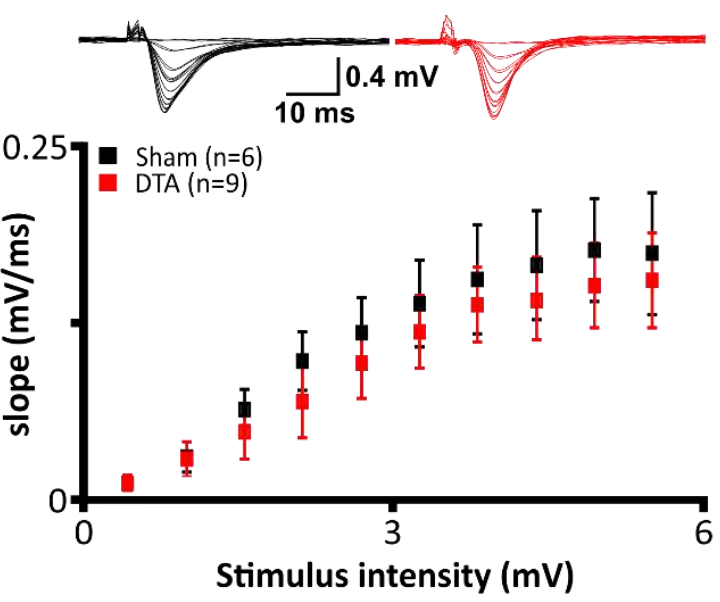

C
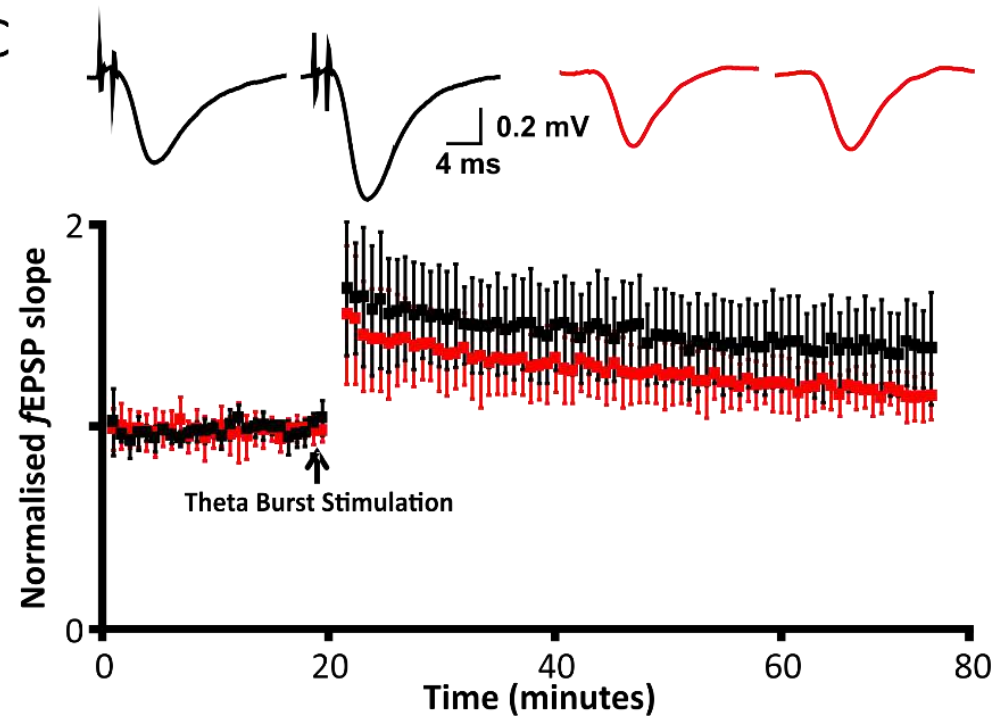

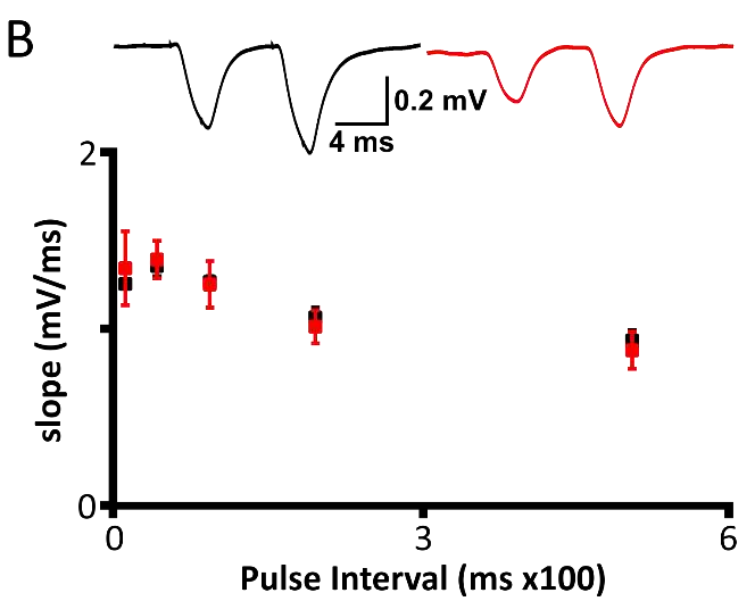

D

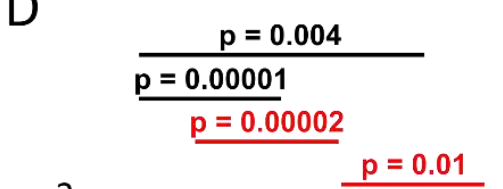

Fig. 5: Induction of SA has no effect on basal transmission but leads to a loss of LTP. A) Stimulus Input-out curves for SA and sham mice. Top) representative data from individual rats, and Bottom) group data. There is no significant difference between the two. B) Paired pulse facilitation was also unaffected. Top) representative data from individual rats, and Bottom) group data. There is no significant difference between the two. C) Average recordings of the slope of EPSPs from electrophysiological recordings show LTP is diminished in rats with SA (red), but not shams (black), following theta burst stimulation (TBS). Top) representative data from individual rats, and Bottom) and sham rats. Data represented as mean \pm SD. 
A
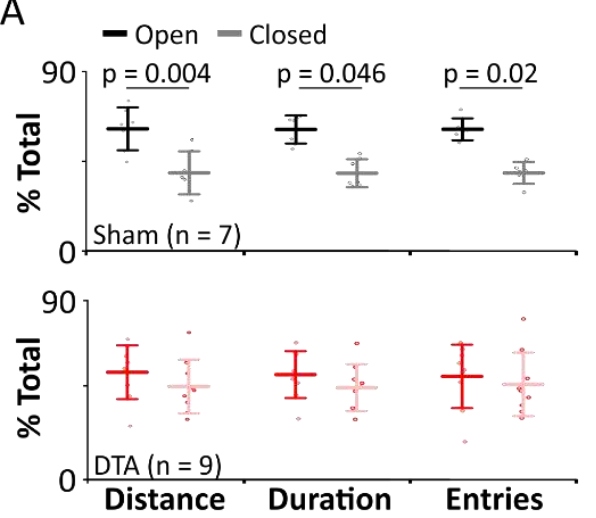

B

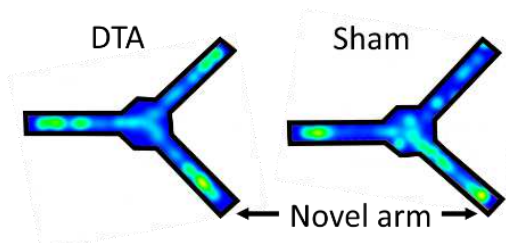

C
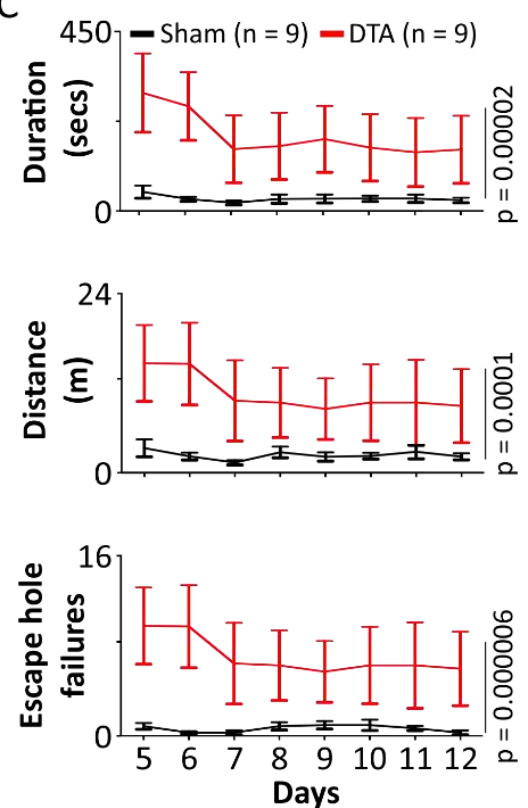

D
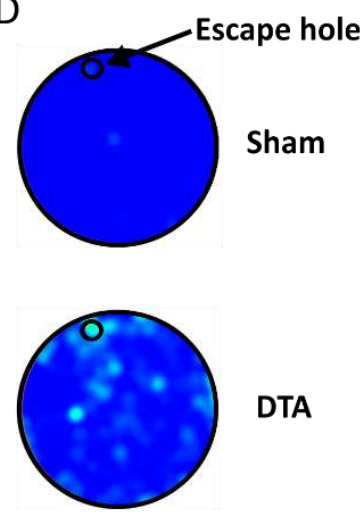

DTA

$\mathrm{E}$

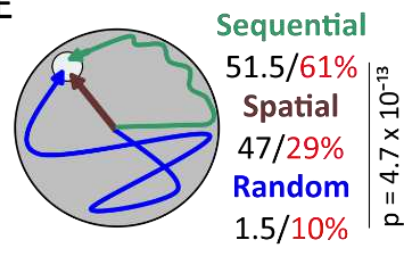

Fig. 6: Our model of SA exhibits cognitive decline. A) Y-maze. Sham rats (black) spend more time investigating the novel arm (solid box) than the previously open arm (open box); in terms of number of entries, distance covered and time spent in the novel arm. DTA injected rats spend equal time in both arms. B) Heat maps from representative rats. C) Rats with SA (red) take longer to complete the maze and cover more distance on the maze than sham operated rats (Black). D) Heat maps from individual rats. E) Cartoon displays search strategies used by sham and SA rats; SA rats are less likely to use spatial clues to find the escape hole than sham controls. 


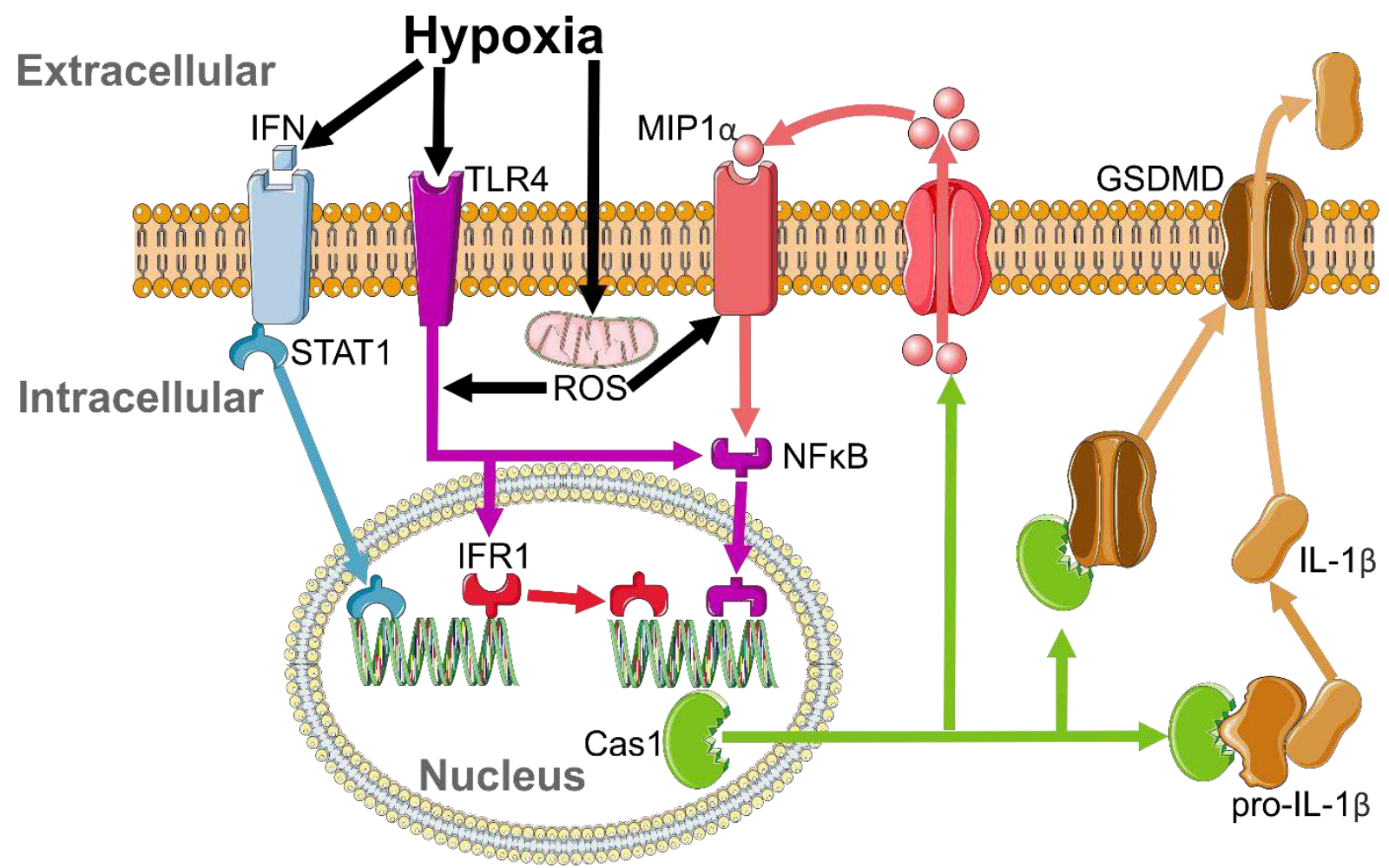

Fig. 7: Proposed inflammatory cascade in sterile inflammation in sleep apnoea. IFNY is elevated by hypoxia and activates interferon regulatory factor 1 (IRF1) through stimulation of STAT1. IFR1 acts as a transcription factor that drives the production of caspase 1 . Activation of the MIP1 $\alpha$ R and the toll like receptor 4 (TLR4) pathway by reactive oxygen species (ROS) released from mitochondria during hypoxia, both enhance Capase 1 production through activation of NFKB. Caspase 1: cleaves pro-IL-1 $\beta$ to release it's active form, IL-1 $\beta$; leads to the insertion of GSDMD, which allow for the translocation of $\mathrm{IL}-1 \beta$ from the intracellular to extracellular space; and increases MIP1 $\alpha$ release. 
A

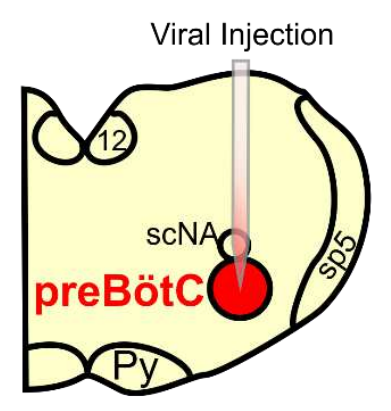

D

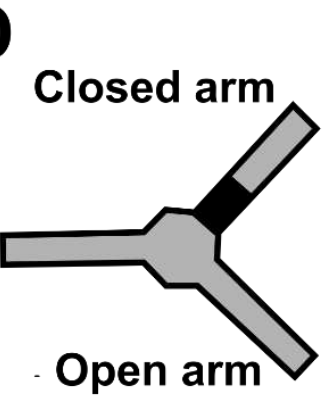

B

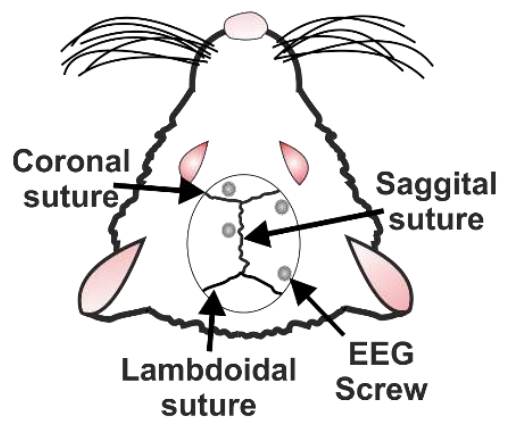

E

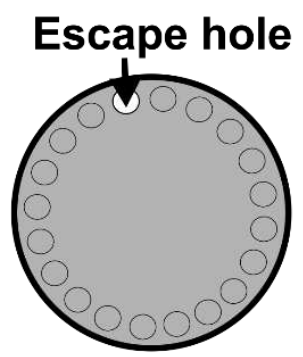

C

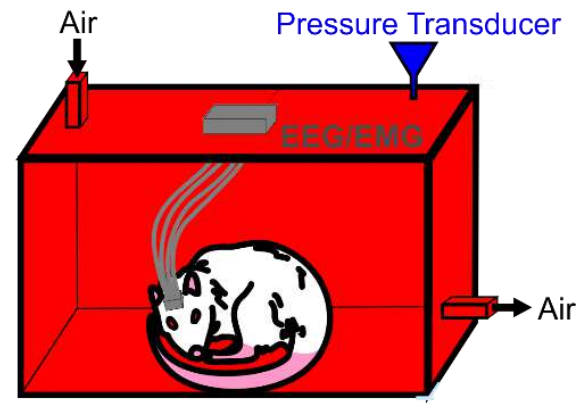

F

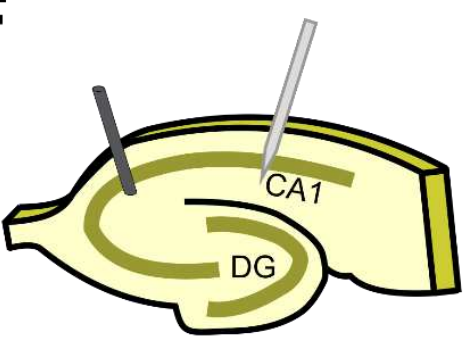

TBS

G

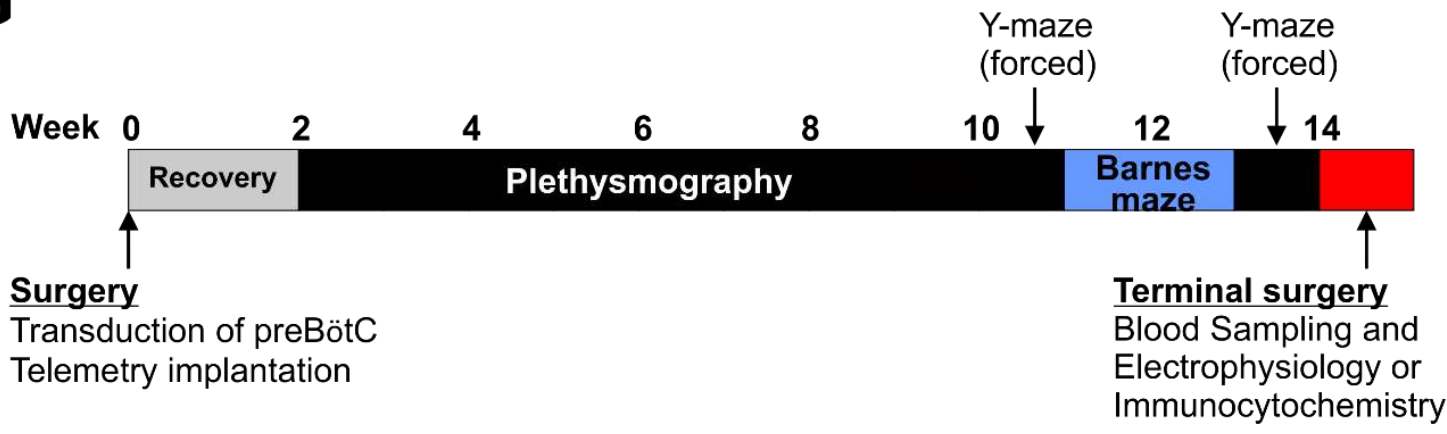

Fig. 8: Creating an testing a new model of SA. A) Sterotaxic Injection of AAV9: syn-DTA-GFP into the preBötC was used to create a stable lesion of the inspiratory oscillator. B) EEG and EMG electrodes were used to determine sleep-wake states. C) Rats were placed into a custom built 4.9L plethysmographic chamber to record breathing and EEG and EMG signals to identify sleep apnoeas. D) A Y-maze was used to determine short-term memory. E) A Barne's maze with 22 escape holes was used to test long term memory. F) LTP recordings were made to test for neurophysiological changes in the hippocampus. G) A timeline of the experimental paradigm 